\title{
On the Potential of Fuzzy Logic for Solving the Challenges of Cooperative Multi-Robotic Wireless Sensor Networks
}

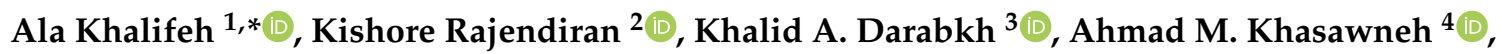 \\ Omar AlMomani ${ }^{5}$ (D) and Zinon Zinonos ${ }^{6}$ (D) \\ 1 Electrical and Communication Engineering Department, German Jordanian University, \\ Amman 35247, Jordan \\ 2 Department of ECE, Sri Sivasubramaniya Nadar College of Engineering, Chennai 603110, Tamilnadu, India; \\ kishorer@ssn.edu.in \\ 3 Computer Engineering Department, The University of Jordan, Amman 11942, Jordan; k.darabkeh@ju.edu.jo \\ 4 Mobile Computing Department, Amman Arab University, Amman 11953, Jordan; a.khasawneh@aau.edu.jo \\ 5 Network Computer and Information Systems Department, The World Islamic Sciences and Education \\ University, Amman 11947, Jordan; omar.almomani@wise.edu.jo \\ 6 Department of Computer Science, Neapolis University Paphos, 8042 Paphos, Cyprus; \\ zinon.zinonos@nup.ac.cy \\ * Correspondence: ala.khalifeh@gju.edu.jo
}

Received: 3 October 2019; Accepted: 27 November 2019; Published: 10 December 2019

\begin{abstract}
Wireless sensor networks have recently been widely used in several applications and scenarios, especially because they have the ability and flexibility for establishing a scalable and reliable wireless network. Cooperative multi-robotic systems (CMRS) are one example of these applications where establishing a wireless network between robots is essential and paramount to their operation. Further, these robots can utilize their mobility to provide sensing functionality for areas that are not covered by the static sensor. This can be achieved by equipping the robots with specific sensors to sense the area of interest (AoI) and report the sensed data to a remote monitoring center for further processing and decision-making. However, the nodes that form the sensor network have limited energy, and, as such, efficient algorithms in clusters' formation, packets' routing, and energy and mobility management are paramount. In this paper, a literature survey is presented containing the most related works that have been proposed to solve these challenges utilizing fuzzy logic. Most of the literature work attempted to utilize a de-centralized approach, where certain input parameters such as the residual energy, communication link quality, network congestion status, the nodes' distance to the sink node and its location with respect to the other nodes, and the data and their sampling rate are all used as inputs to the fuzzy logic controller. These input parameters are used to determine several performance vital factors such as the cluster formation and its cluster head, best route to the sink node, optimal power management policies in terms of sleep/awake times needed to maximize the network lifetime, nodes' mobility management policies to maintain network connectivity, and best route in terms of packet loss and delay.
\end{abstract}

Keywords: fuzzy logic; wireless sensor networks; clustering; routing; power management

\section{Introduction}

A wireless sensor network (WSN) comprises spatially circulated self-ruling sensors to agreeably screen and monitor physical or ecological conditions, out of which contain vibration, temperature, weight, sound, movement, or contaminations [1-3]. It presents a new generation of real-time embedded 
systems, which are characterized by having limited computation, energy, and memory resources. Several applications and scenarios, such as military and remote sensing applications, are roused by WSNs. Further, the improvement of WSNs, for example, in battlefield reconnaissance [4], is currently being utilized in numerous modern and regular citizen application territories, including mechanical or industrial procedure checking and control, machine wellbeing observation, condition and territory checking, medicinal service applications, home robotization, and traffic control [5-7]. It is worth mentioning that every sensor node in a certain network is commonly outfitted with a radio transceiver (or an equivalent wireless communications device); an energy source, usually known as a battery; as well as a micro-controller [8]. Another potential application where WSNs play a crucial role is in cooperative multi-robotic systems (CMRS) [9-14], where several mobile robots cooperate on performing a specific mission within certain area of interest (AoI). For instance, the robots can utilize their mobility to provide sensing functionality for areas that are not covered or reachable by static sensors, thus enhancing the sensing and coverage capabilities of the legacy static sensor network. However, these robots usually communicate with each other, as well as with the base station, in order to have full coordination and optimal management while performing their missions and to send the sensed data to the monitoring center for further processing and decision-making. However, in order to have efficient, reliable, and scalable WSN, several parameters and configurations should be optimized, especially with the mobility challenges of the mobile robots, as well as the limited energy of the sensor nodes. Another type of sensor network is a hybrid network, where static, mobile, and flying nodes are utilized to collaboratively sense the AoI in a comprehensive and efficient manner $[15,16]$.

In order to utilize the limited energy source, in cooperative multi-robotic wireless sensor networks (CMR-WSNs, the sensor nodes are normally grouped into clusters, where all the nodes send their captured data to a central node called a cluster head $(\mathrm{CH})$. This not only saves energy, but also mitigates interferences between the different nodes that are deployed within certain AoI. Typically, data is sent to a remote monitoring center for further analysis and decision-making. Therefore, $\mathrm{CHs}$ communicate with each other and send the captured data using multi-hop routing until the data reaches the remote and decision-making destination center. Both clustering and routing processes are vital in WSN operation and need to be optimized in order to increase the network lifetime. Generally, and as indicated by the WSN structure, routing conventions can be grouped mainly into flat-based and clustering-based protocols [3-6]. Clustering-based routing conventions are the most widely recognized method that is utilized in WSNs. It is critical to refer to the fact that a massive measure of research on WSNs is coordinated towards expanding the network lifetime by making them energy proficient [3-8]. The clustering method is considered as one of the key activities that are actively investigated for prolonging the network lifetime in WSNs $[7,8,17,18]$.

Furthermore, optimizing the nodes' power management in terms of sleep/wake times is very crucial to achieve an extended life network, especially in that the nodes are normally deployed in an area that is difficult to reach, and, as such, recharging their batteries may not be a viable option. In addition to these challenges, mobility management is another challenge that is associated with mobile sensor nodes that are witnessing increased growth in usage and adoption in several applications and scenarios [4]. The existence of mobile nodes creates some new challenges in wireless sensor networks such as power consumption, dynamic network topology, mobility of the sink, and localization. The new challenges are strongly connected to a critical issue of mobile sensor networks, that is, network connectivity.

Artificial intelligence mechanisms have been utilized in previous WSN research, particularly in routing protocols; clustering algorithms; and power management including fuzzy logic [19], artificial neural network (ANN) [20], neural fuzzy inference system (ANFIS) [21], and optimization algorithms, such as particle swarm optimization (PSO) [22], genetic algorithms [23], gravitational search algorithm (GSA) [24], and bacterial foraging algorithm (BFA) [25]. The innovation of fuzzy and neuro-fuzzy systems in WSNs utilizes soft computing concepts to not only deal with the increment execution of WSNs, but also make them increasingly intelligent. Interestingly, fuzzy logic helps in settling on constant choices without requiring information about the environment [26]. Then again, regular 
control instruments or approaches, for the most part, need precise and complete knowledge about the environment. Fuzzy logic can likewise be used for settling on a choice (i.e., making a decision), which is dependent on various environmental parameters that are required to be combined as indicated by predefined rules $[27,28]$. In particular, fuzzy logic is utilized in clustering for blending distinctive clustering parameters, as indicated by predefined standards or rules and, afterward, choosing cluster heads is dependent on the outcomes acquired. Moreover, as widely reported in the literature, the cluster head election overhead is expected to be substantially reduced through the efficient use of fuzzy logic.

The aim of this paper was to highlight the main contributions that utilize fuzzy logic in addressing the major challenges of CMR-WSNs, which were used as a base for the conducted literature review and selected papers. The main contributions of this paper can be summarized as follows:

- Identification of the main challenges for WSN used in cooperative multi-robotic scenarios, namely, clustering, routing, power management, and mobility management.

- Conducting a comprehensive literature review for the papers that propose fuzzy logic-based solutions to the CMR-WSNs' main challenges and providing comparison tables for various fuzzy-based approach solutions and algorithms discussed in the literature.

In the literature, several works describe different fuzzy-based solutions for tackling CMR-WSNs' challenges. However, there are very few papers that group these solutions together, summarize them, as well as conduct comparisons between them.

The remainder of the paper is organized as follows. Section 2 provides a general technical overview of fuzzy logic. Section 3 summarizes the most recent related works for solving the clustering problem utilizing fuzzy logic. Section 4 presents the related works that utilize fuzzy logic as a reason for addressing the WSN routing challenge. Section 5 discusses how fuzzy logic can be used in optimizing nodes' power management. Section 6 describes the potential of fuzzy logic in managing mobility for CMR-WSNs. Finally, Section 7 concludes the paper and proposes future research directions.

\section{Fuzzy Logic Overview}

The general structure of a fuzzy logic-based decision system is depicted in Figure 1, which consists of three main building blocks: the fuzzifier, the inference engine, and the de-fuzzifier [21]. The fuzzy logic system (FLS) knowledge is represented by fuzzy variables that can take any values between 0 and 1, as opposed to the binary logic system; further, it compromises a set of IF-THEN rules. To illustrate this, consider an input variable that has an exact value that is measured, for example, from a weather temperature sensor-in fuzzy logic, these exact values are called crisp values. Next, the fuzzifier will group the crisp input values and map them to a fuzzy set using the membership function. The fuzzifier output named the fuzzy linguistic variables are used to describe the system qualitatively. For example, they can describe the weather on the basis of the measured temperature as hot, cold, warm, freezing, and so on.

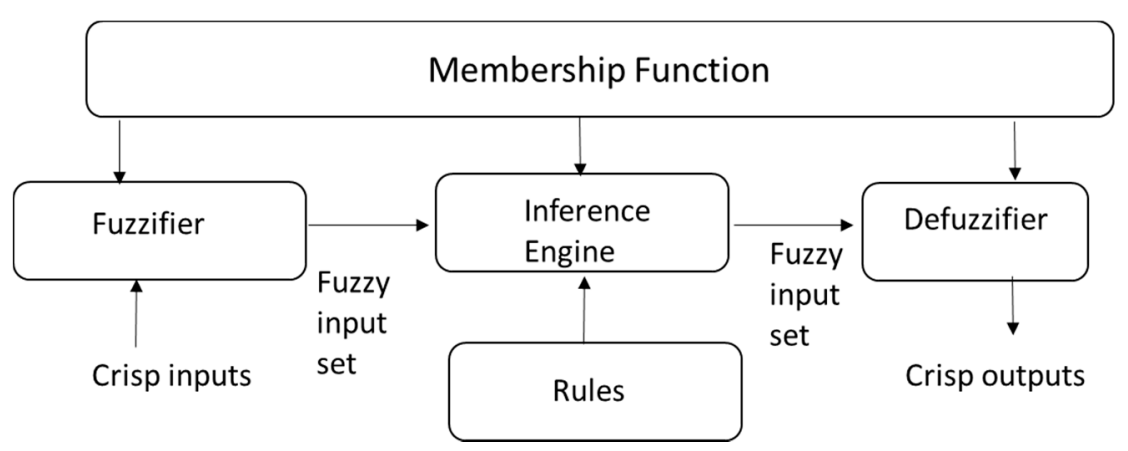

Figure 1. Fuzzy logic system structure [29]. 
After that, the inference engine shown in Figure 2 maps the input values to a certain fuzzy output on thebasis of a set of IF-THEN rules, and the fuzzy outputs of these rules are then aggregated using the aggregator function. Aggregation is the process by which several fuzzy values are mapped to a single fuzzy set that represents the output rules of the fuzzy rules. For example, the weighted means function can be used as an aggregator to map several fuzzy values to a single value that will be used in the de-fuzzification process. Finally, to get a deterministic decision from the fuzzy logic system, the aggregator output is fed to the de-fuzzification process, which is responsible for getting a crisp output (non-fuzzy output) using membership functions analogous to the one used in the Fuzzifier stage. The choice of the appropriate de-fuzzifier function is application-specific. However, centroid of area (COA); bisector of area (BOA); and the mean, smallest, and largest of maximum (MOM, SOM, $\mathrm{LOM}$, respectively) are the most commonly used functions [30].

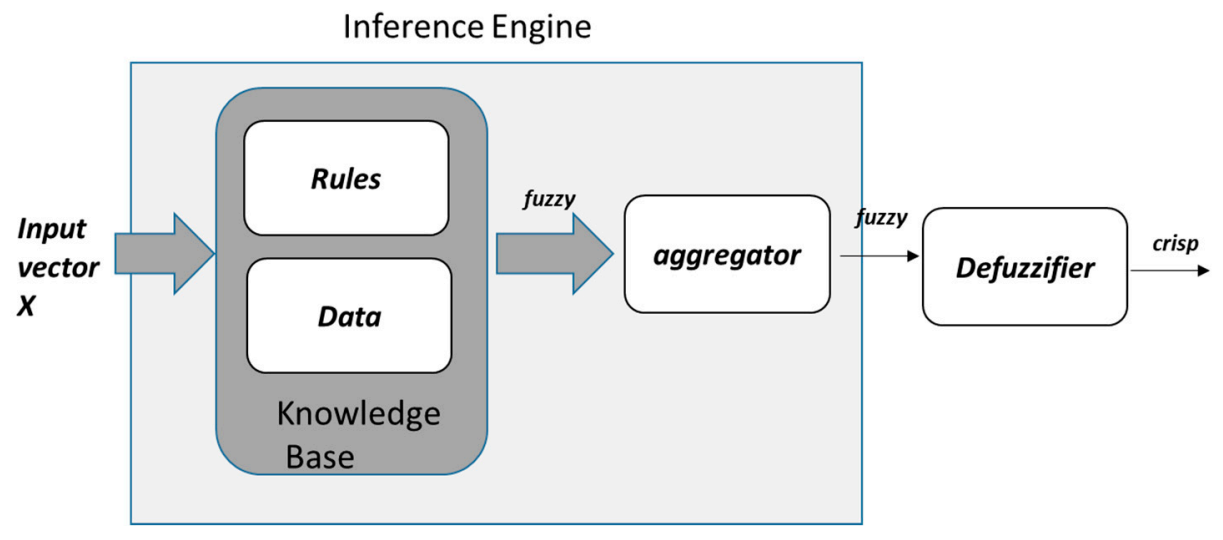

Figure 2. Fuzzy logic interference engine rules.

\section{Fuzzy Logic-Based WSN Clustering}

In WSN, nodes clustering and cluster head election play a vital role in optimizing the network operation and prolonging its lifetime. As depicted in Figure 3, sensor nodes (SNs) are usually distributed in an AoI to sense and cover it; each node should send its sensed data to the base station (BS), where data is processed and analyzed to evaluate the situation and make a proper decision if needed. For instance, if these nodes reported an abnormal increase in $\mathrm{CO}_{2}$ gas concentration, then this may be an indicator of having a fire, for example, where immediate action should be taken (e.g., sending firefighters to the monitored area). If the deployed sensor nodes communicated their sensed data directly to the BS, each node has to use high power to compensate for the path loss due to the long distance between them and the BS. Further, SNs will interfere with each other, and the BS will incur a high packet loss rate. To solve this issue, nodes are normally clustered, where a $\mathrm{CH}$ node is selected, and all the $\mathrm{SNs}$ that are close to the $\mathrm{CH}$ node will form a cluster and communicate their captured data to the $\mathrm{CH}$, which in turn will forward it to the BS. This scheme minimizes the energy consumed in transmitting the data, as SNs have to send their data to the $\mathrm{CH}$, which is closer and needs much less power than communicating directly with the BS. Further, SNs within the cluster can utilize a specific communication channel that will not interfere with other nodes in the other clusters. It is noticeable that the way clusters are formed will significantly affect performance. In what follows, a literature review for the most related work that utilizes fuzzy logic approach in establishing the network clusters and cluster head selection is presented. 


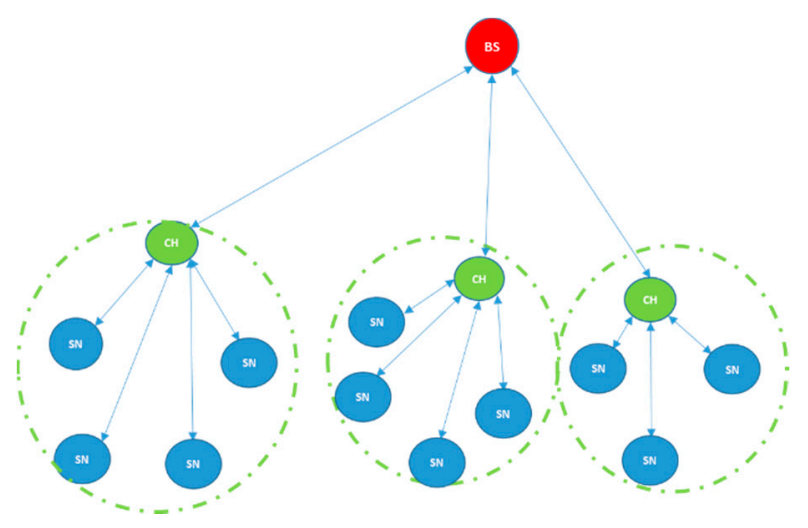

Figure 3. Sensor nodes divided into three clusters, where each cluster has a cluster head that sends the captured data to the base station for further processing and analysis.

Kim et al. [31] proposed a cluster head election mechanism using fuzzy logic (CHEF). In the traditional clustering models such as the low energy adaptive clustering hierarchy (LEACH) [32], all the sensor nodes get a chance to become cluster head on the basis of a probabilistic model, evenly distributing the energy consumption. However, in such a scenario, the elected cluster heads may be very close to each other, and in few cases, the cluster heads are located on the edge of the cell because the election process is purely based on a probabilistic model. Moreover, LEACH does not consider the existing battery power of the nodes during the cluster head selection process, which may lead to the selection of nodes with relatively small battery power. Such limitations motivated the research on clustering mechanisms based on fuzzy logic. In the fuzzy logic-based mechanisms discussed in the literature, parameters such as concentration, energy, and centrality were used as fuzzy variables to enhance the overall lifetime of the network. Such fuzzy-based schemes are centralized, wherein all the required information from all the nodes are collected at the base station, and, consequently, the cluster head is selected on the basis of the collected information using fuzzy logic. However, to avoid having a centralized solution, Kim et al. [31] proposed CHEF where the election is based on IF-THEN fuzzy rules, and the operation is localized. Here, the base station is not involved in cluster head election and the sensor nodes take the responsibility to elect the cluster head using fuzzy rules. On every round, the nodes generate a random number between 0 and 1 . If the number is less than the predefined optimum value, the node then computes the chance of being a cluster head using a fuzzy IF-THEN rule. Here, the authors introduced fuzzy variables such as energy, local distance (sum of the distances between the nodes), and the nodes' density. The sensor node calculates a chance from its remaining battery power, the local distance, which is the sum of distances between itself and other nodes within $r$ distance. The bigger the chance, the higher the opportunity of becoming a cluster head. The node then advertises a CANDIDATE message with the chance value and likewise receives CANDIDATE messages from all other nodes of the network. If the chance value in and of itself is bigger than the other nodes, then the node announces itself as a cluster head and advertises CH_message. The nodes that are not cluster heads acknowledge the $\mathrm{CH}$ _message with Cluster_Join message. In this work, the authors have used de-fuzzification to transform the fuzzy variables into a single crisp value. The authors showed that any two cluster heads cannot exist within a distance $r$, and more efficient cluster heads were elected as energy and distance were considered in the selection process. Further, the overhead calculation was reduced due to fuzzy logic and, finally, the network lifetime was enhanced.

Hamzah et al. [33] proposed a fuzzy logic-based energy-efficient clustering for wireless sensor networks on the basis of minimum separation distance imposition between cluster heads. In this work, the authors emphasized the fact that if the clustering algorithm exploits more energy affecting factors, then the clustering will be more efficient. The fuzzy inference system is used for obtaining better parameter integration. The presented work uses five parameters to elect the cluster head. The parameters are the residual energy, location suitability, density, compacting, and distance from the base 
station. On the basis of these five descriptors, cluster heads are selected, and the remaining non-cluster head nodes join one of the elected cluster heads. The authors set a minimum distance of separation between any two cluster heads to ensure even distribution. In addition, the authors used the Gini index to measure the energy efficiency of clustering algorithms in terms of their ability to balance the distribution of energy through the nodes. The performance of the presented work for various network densities and scenarios is compared with the existing methods. The authors showed that the proposed approach outperformed the existing work in terms of energy efficiency and network lifetime.

Thangaramya et al. [34] proposed a neuro-fuzzy rule-based cluster formation and routing protocol for efficient routing in IoT-based WSNs. The authors identified that in most of the fuzzy-based clustering protocols available in the literature, the cluster size is not considered, which may lead to non-uniform energy consumption. Most of the work in the literature addressed fuzzy rule-based clustering for stationary nodes and used a probabilistic approach for decision-making. To overcome these limitations, the authors used parameters such as cluster head energy, the distance between cluster head and base station, change in the distance between cluster head and member nodes due to mobility, and cluster head degree as inputs to the fuzzy inference system. In the proposed protocol, the network is trained and tested utilizing a convolutional neural network with a neuro-fuzzy rule manager for weight adjustment. In this work, the base station sent HELLO packets to all the nodes, and the distance between the nodes and base station was computed. Clusters were formed using $k$ means clustering algorithm and the base station performed cluster head selection by considering the distances and nodes' energy levels. The authors used a fuzzy reasoning approach for proper cluster formation followed by efficient routing.

Further, the authors adopted the Mamdani inference system in making a decision. De-fuzzification mapped the fuzzy set to a crisp set for exact decision-making. Once the cluster head was elected, the nearby nodes had to decide to join one of the elected cluster heads. In this process, the fuzzy inference system used the four input parameters as mentioned earlier. Once the cluster head was elected, the best route was decided by finding the shortest route between each node and the base station through the cluster heads. The authors inferred that the presented work performed better compared to the existing techniques in the literature in terms of energy utilization, packet delivery ratio, delay, and network lifetime. The limitation of the presented work was that all the nodes were assumed to be trustful nodes, which might not have been the case.

Nguyen et al. [35] proposed a novel clustering scheme for WSN based on fuzzy logic to improve the lifetime of the network. In this work, the authors used parameters such as residual energy, distance from the sink, and the density of the nodes in a locality as the inputs to the fuzzy system. Once the sensor nodes were deployed in the area of interest, the nodes self-configured themselves as a network. In this work, there were two essential phases, namely, cluster head selection and formation. The cluster head was elected based on fuzzy rules. The authors used a fuzzy inference system as the decision-making unit that used IF-THEN rules along with OR/AND connectors for decision making. This decision-making behavior was effectively handled by computing the rank of each node. The parameters such as density, remnant energy, and distance from the base station were applied as inputs to the fuzzy inference system. The overhead of the cluster head was also considered during the cluster head selection process. Further, similar to the work presented in [34], the authors adopted the Mamdani inference system that imitated the human inference system in making a decision. De-fuzzification mapped the fuzzy set to a crisp set for exact decision-making. Once the cluster head was elected, the nearby nodes had to decide to join one of the elected cluster heads. In this process, the fuzzy inference system used three input parameters such as density, closeness to the cluster head, and cluster head residual energy. A node expressed its willingness to join a cluster by sending a 'JOINING' request packet to the cluster head, and the request would then be acknowledged by the cluster head. Once the clusters were formed, the cluster head shared a time division multiple access (TDMA) slot with all the member nodes in order to avoid a collision. The collected data were fused and transferred to the base station for further processing by the end-user. The authors inferred that the performance in terms of 
stability period and protracted lifetime was better when compared to the traditional schemes such as LEACH and CHEF discussed in the literature.

Nayak et al. [36] proposed a novel clustering algorithm for wireless sensor networks to extend the lifetime of the network. In this work, the authors attempted to improve the performance of the LEACH protocol by introducing the concept of super cluster head (SCH) election. SCH was elected among the cluster heads by applying appropriate fuzzy descriptors, and only the $\mathrm{SCH}$ could communicate with the base station. The authors showed that the proposed technique reduced the number of transmissions and retransmissions performed by the cluster heads. In this work, the authors used parameters such as remaining battery power, mobility, and centrality as the fuzzy descriptors to elect an appropriate $\mathrm{SCH}$ that can communicate with the base station. Because the energy level of the cluster head gets reduced each round, remaining battery power was considered as one of the metrics. Another critical metric was centrality, which depicted the reachability of $\mathrm{SCH}$ concerning all the cluster heads, and the mobility metric referred to the variation in the distance between the base station and $\mathrm{SCH}$, as the authors assumed that base station was mobile. Here, the proposed algorithm was composed of four essential blocks, these being fuzzification, rule evaluation, fuzzy inference engine, and de-fuzzification. In this work, the authors used the Mamdani's fuzzy inference technique to elect an appropriate SCH, which used the metrics mentioned above as inputs. Here, the authors chose the linguistic variables on the basis of fuzzy descriptors. Less, medium, and high were used as linguistic variables for remaining battery power; low, moderate, and frequent were used for mobility; close, adequate, and far were used for centrality. The authors inferred that the performance in terms of overall network lifetime was better when compared to the traditional schemes discussed in the literature due to the idea of sink mobility along with the fuzzy logic-based election of SCHs.

Most of the research works reported in the wireless sensor networks domain considered random deployment of nodes. In such cases, there was a possibility that the nodes were not deployed uniformly in the area of interest, which may have led to network partition or formation of holes in the network. In such scenarios, there is a possibility that the nodes in the edges of the holes may not capture the event and report it to the cluster head in the correct time, which may lead to a huge disaster. Vajdi et al. [37] proposed an approach to detect the holes in the network. Here, the authors used the Delaunay triangulation approach to detect the holes in the network and applied a virtual gridding scheme to form grids that represent a cluster. The authors inferred that the employed gridding mechanism saved a considerable amount of energy and time compared to the traditional clustering mechanisms discussed in the literature. In this work, the authors proposed a novel idea of constructing routing paths, namely, common and emergency routing paths. Common routing paths were used by the cluster heads that do not use their full signal strength to transfer messages to the base station, whereas hop nodes that use their full signal strength to transfer critical messages to the base station used the emergency routing paths. Finally, cluster heads and hop nodes were elected by employing fuzzy logic-based approaches to overcome the uncertainties in the environment. In this work, the authors used residual energy and centrality as the fuzzy descriptors for electing the cluster heads, whereas residual energy and radio energy dissipation were used as fuzzy descriptors for electing hop nodes. The authors showed that the proposed mechanism was efficient and applicable to event-based large-scale wireless sensor networks, as the proposed model was not based on rounds and did not stop the network's operation for reconfiguration.

In the traditional clustering approaches discussed in the literature, clusters were formed, and cluster heads were elected in more or less the same way. However, practically, the same node cannot continue as a cluster head for a longer duration, which may lead to battery exhaust and, hence, cluster reformation was proposed, wherein the protocol should reform clusters and rotate the responsibility of the cluster heads among the member nodes to improve the network lifetime. Although these protocols were best suited for clustering, it was proven that these protocols do not guarantee suitable cluster head selection. To overcome these limitations, Toloueiashtian et al. [38] proposed an energy-efficient clustering protocol using fuzzy logic utilizing fuzzy parameters such as the amount of energy in cluster 
head, distance from cluster head to the base station, and the number of connections in cluster head. In this work, the member nodes were attached to a cluster head having a higher chance concerning the aforementioned three parameters and the fuzzy inference system. For example, if the energy in the cluster head was low, the distance from the base station to the cluster head and the number of connections to the cluster head was high, and then automatically the chance of becoming a cluster head became very low. On the other hand, if the energy level in the cluster head was high, the distance from the base station to the cluster head and the number of connections to the cluster head was low, and then the chance of this node to be a cluster head became very high. The authors depicted that the proposed algorithm achieved an improvement of about $12 \%$ in the network lifetime compared with LEACH [24], and the fuzzy logic cluster formation protocol (FLCFP).

Qin et al. [39] introduced a synchronous distributed fuzzy C-means algorithm that was capable of clustering the nodes based on the nodes' measured data, with degrees of membership values varying from 0 to 1 . Simulation results showed that the decentralized approach had almost similar results to the centralized one. However, as expected, by de-centralized algorithms, they had higher communication overhead in terms of data exchanged between nodes than centralized algorithms. Recently, the authors in [40] reported a distributed C-means clustering algorithm implementation in an asynchronous distributed fashion via broadcast-only token-passing for wireless sensor networks to address the issues such as managing a huge amount of data that were collected from the field of interest and analyzing the behavior of the nodes on the basis of the collected data. In this work, the authors proposed to partition the networks into homogeneous groups, such that the agents belonging to the same set had similar information, and agents in different groups would have distinct information. The presented work organized data transmission following a broadcast only token-passing approach, thereby reducing the undesirable communication efforts that may happen in such networking scenarios. The simulation results depicted that the distributed C-means algorithm based on asynchronous, event-driven, and broadcast-only communication yielded similar results to the centralized C-means algorithm, but with a reduced amount of data exchanged compared to synchronous distributed fuzzy C-means algorithm [39].

In the study by [41], the authors proposed a distributed opinion dynamics model utilizing fuzzy states. This model was extended and used to cluster wireless sensor nodes in a distributed manner [42], where each sensor held a large set of data. The main goal was to group the nodes into homogeneous clusters by information type. In the literature, most of the proposed algorithms ask the user to specify the desired number of clusters beforehand. In their approach, the number of clusters was calculated on the basis of the distance constraint among cluster centroids, which did not require a central authority and depended only on the exchanged data between nodes, thus making the clustering algorithm rely merely on the sensed data and not on the network topology.

Figure 4 depicts a diagram summarizing the different parameters discussed in this paper used in cluster formation utilizing fuzzy logic.

Table 1 summarizes the various protocols discussed earlier in terms of some essential characteristics and capabilities possessed. All the protocols discussed in Table 1 are cluster-based protocols implemented with the fuzzy-based approach. According to Table 1, self-management model [37] performs better than the other mechanisms as it is highly reliable because the authors proposed a novel hole detection mechanism along with the fuzzy-based approach. One more vital factor to be noted in the comparison chart is that the self-management model is not round-based, and hence the network operation is never affected due to reconfiguration. In addition, the model proposes the idea of hop nodes, which, in case of emergencies, can deliver critical information faster to the base station. 


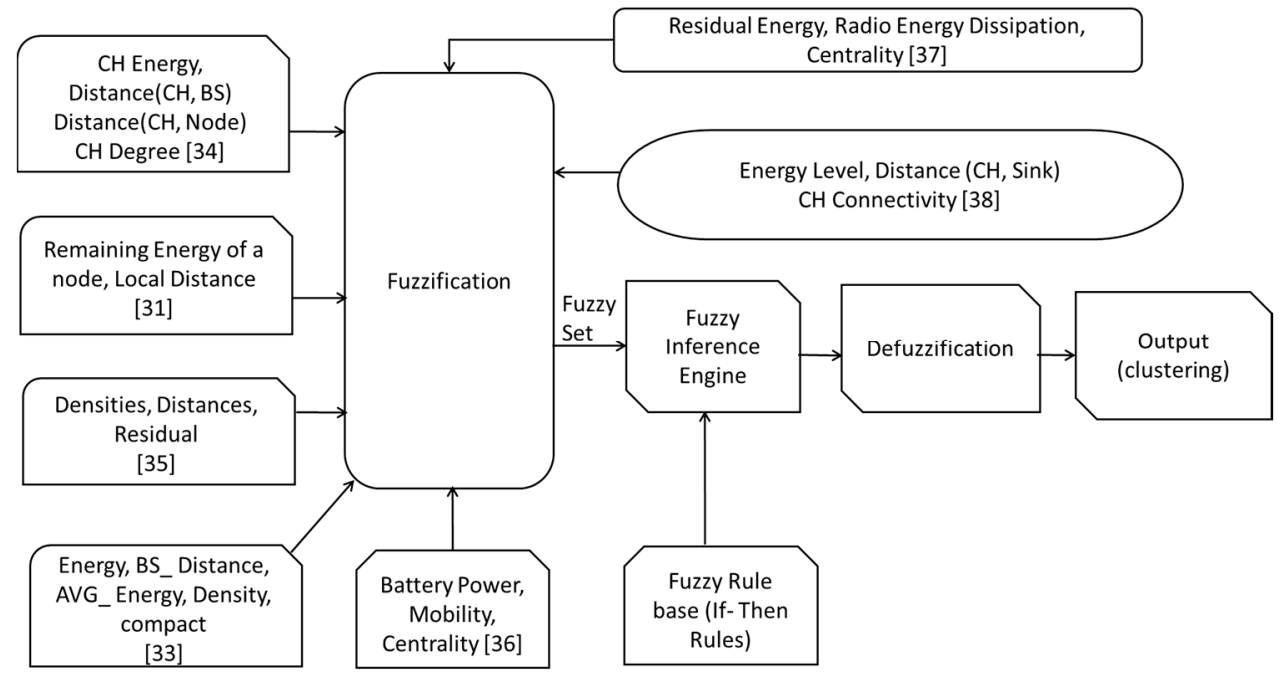

Figure 4. A block diagram summarizing the different parameters discussed in this paper used in cluster formation utilizing fuzzy logic. $\mathrm{CH}$ : cluster head, BS: base station.

Table 1. A comparison for the surveyed fuzzy-based clustering protocols.

\begin{tabular}{|c|c|c|c|c|c|}
\hline Protocol/Algorithm & Round-Based & Cluster Size & Fuzzy-Based & Multi-Hop & Detecting Hole \\
\hline $\begin{array}{l}\text { Cluster head election } \\
\text { mechanism using } \\
\text { fuzzy logic [31] }\end{array}$ & Yes & Not fixed & Yes & No & No \\
\hline $\begin{array}{l}\text { Fuzzy logic-based } \\
\text { energy-efficient } \\
\text { clustering for WSN on } \\
\text { the basis of minimum } \\
\text { separation distance } \\
\text { imposition between } \\
\text { cluster heads [33] }\end{array}$ & Yes & Not fixed & Yes & No & No \\
\hline $\begin{array}{l}\text { Energy aware cluster } \\
\text { and neuro fuzzy-based } \\
\text { routing algorithm, [34] }\end{array}$ & Yes & Fixed & Yes & No & No \\
\hline $\begin{array}{c}\text { Fuzzy logic for } \\
\text { clustering scheme [35] }\end{array}$ & Yes & Not fixed & Yes & No & No \\
\hline $\begin{array}{l}\text { Fuzzy logic-based } \\
\text { clustering algorithm } \\
\text { for wireless sensor } \\
\text { network [36] }\end{array}$ & Yes & Fixed & Yes & No & No \\
\hline $\begin{array}{l}\text { Self-management } \\
\text { model [37] }\end{array}$ & No & Fixed & Yes & Yes & Yes \\
\hline $\begin{array}{l}\text { Clustering approach in } \\
\text { WSN using fuzzy } \\
\text { system [38] }\end{array}$ & Yes & Not fixed & Yes & No & No \\
\hline
\end{tabular}

Furthermore, in most of the fuzzy-based clustering algorithms discussed in the literature, the metrics such as first node dies (FND), half of the nodes dies (HND), and last node dies (LND) were used to evaluate the network lifetime [33,35,37]. The authors validated the performance of the proposed techniques under different network densities and for different locations of base station. Table 2 depicts the network lifetime in rounds based on the HND metric. Average lifetime in rounds was calculated using the fuzzy descriptors proposed by the corresponding authors. The projected data demonstrate the variations in the performance on the basis of the fuzzy descriptors utilized in the fuzzy-based clustering algorithms. 
Table 2. Half of the nodes dies (HND) comparison.

\begin{tabular}{ccc}
\hline Fuzzy-Based Clustering Algorithms & Fuzzy Descriptors & Average Lifetime in Rounds \\
\hline CHEF [31] & $\begin{array}{c}\text { remaining energy of a node, local distance } \\
\text { energy, BS_Distance, AVG_Energy, } \\
\text { density, compact } \\
\text { FL-EEC/D [33] }\end{array}$ & 630 \\
CH energy, \\
$\begin{array}{c}\text { Energy aware cluster and neuro fuzzy } \\
\text { based routing algorithm, FBCFP [34] }\end{array}$ & $\begin{array}{c}1437 \\
\text { distance (CH, BS) } \\
\text { Fuzzy logic for clustering scheme [35] } \\
\text { Fuzzy logic-based clustering } \\
\text { algorithm for WSN [36] }\end{array}$ & CH degree \\
Self-management model [37] & densities, distances, residual & 1400 \\
battery power, mobility, centrality & 675 \\
\hline
\end{tabular}

\section{Fuzzy Logic-Based Routing Algorithms}

This section reviews the-state-of-the-art routing algorithms that use fuzzy logic systems for selecting the next forwarding nodes among candidate sensor nodes. This section highlights the main parameters utilized by each algorithm with different fuzzy logic systems. All of the presented algorithms focus on forwarding strategies by selecting the node that is placed closer to the sink node. In fact, the improper selection of the next forwarding node can be considered as one of the main problems in routing protocols that have a direct impact on packets' delivery. The selection of the next forwarding nodes with high residual energy, best link quality, and physical distance helps in reducing packet loss, leading to increase reliability, energy-efficiency, and reducing delay. Furthermore, proper selection to the next hop reduces interference and can achieve better load balancing between the intermediate nodes in terms of energy consumption and network traffic flows, which is depicted in Figure 5. As shown in Figure 5a, if both nodes 1 and 2 want to send data to node 8, then node one may choose the blue route, and node two may choose the green route which will overland nodes 4 and 6 , as they are relaying the traffic and are thus consuming more energy and reducing their batteries. On the other hand, in Figure 5b, node 1 has chosen a different route, which loads balance the traffic among all nodes, thus not overloading any nodes and obtaining a fair energy consumption among all nodes.

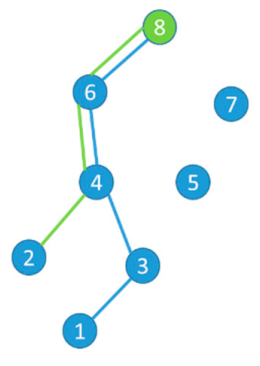

(a)

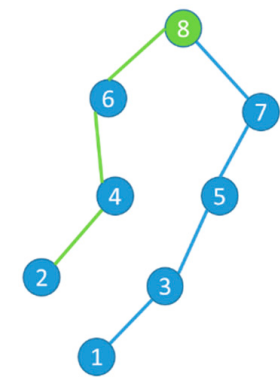

(b)

Figure 5. Depicts the routing paths for the traffic generated by nodes 1 and 2, in green and blue colors, respectively. (a) Demonstration of a possibility for overloading nodes 4 and 6 while routing the traffic to node 8 , where (b) shows a more balanced traffic distribution among the nodes.

To solve these problems, several researchers have introduced several fuzzy-based intelligent delays and energy-aware algorithms. In what follows, a survey of the most known examples is presented. Mothku and Rout [43] proposed a protocol that comprises a fuzzy-based energy-aware routing mechanism (FEARM) and fuzzy logic system. The protocol starts by finding the next forwarding nodes among neighbors by combining four parameters as input values for the FLS, namely, link quality, residual energy, physical distance, and available buffer, as shown in Figure 6. The output of FLS can be considered as a metric value named "chance of becoming the next node". In terms of routing, this 
protocol takes advantage of Dijkstra's algorithm to establish a different route and then discovers the best path. Simulation results show that the proposed protocol reduces packet loss, delay, and energy consumption. Moreover, it proves that the use of fuzzy with four parameters performs better results than fuzzy with fewer parameters.

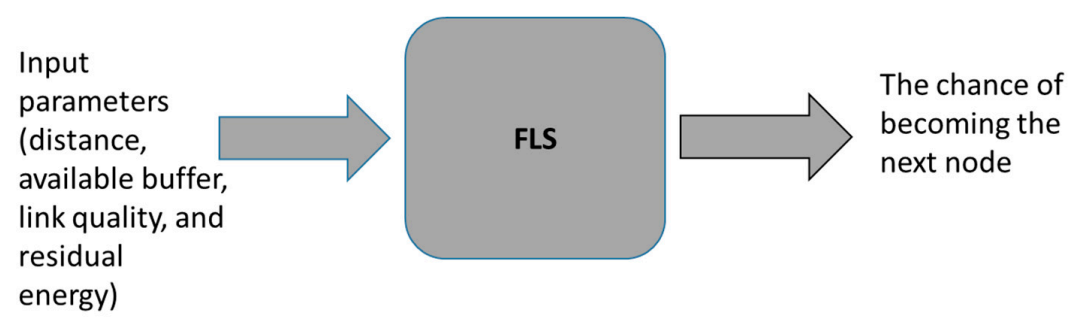

Figure 6. The fuzzy logic system used for routing process.

Hu et al. [44] proposed a novel fuzzy logic-based geographical routing (FLGR) protocol to enhance routing overhead, void, and location accuracy problems. FLGR proposes a novel-forwarding mode based on the fuzzy location region of the receiver node and void avoidance scheme, being a fuzzy logic system that is capable of choosing the routes with a lower number of hops and with high packet delivery ratio. FLGR is composed of three phases-it starts by determining the next forwarding nodes on the basis of the fuzzy location region of the receiver node. Next, the best forwarding node is selected on the basis of the following three steps: fuzzification, fuzzy reasoning, and de-fuzzification of the node's parameter. Lastly, in the routing process, avoid avoidance scheme was designed and developed to avoid the void nodes in trap areas. Simulation results demonstrated that FLGR can avoid the trap area efficiently. Moreover, FLGR performed a high delivery ratio and reduced routing overhead compared with existing protocols.

Gholipour [45] conducted a novel research study focusing on prolonging the network lifetime named "a new ant colony optimization routing approach based on fuzzy clustering". This approach is composed of two phases: clustering and routing. In clustering, a local self-selection process by an ordinary node is applied to be a cluster head, and then the decision among neighbors in the network is advertised. This process is split into two phases: setup and steady. In the first phase, an ordinary node uses a fuzzy system to calculate its chance of helping in deciding to be a cluster head on the basis of residual energy, number of neighbors, received signals, and centrality. The node with a higher chance becomes a cluster head and determines its members.

On the other hand, clusters are fixed until the end of the steady phase. This approach is repeated continuously until the end of the network. In the routing phase, an inter-cluster routing was proposed on the basis of ant colony optimization as per the following steps. First, finding the best relay node for cluster head on the basis of neighbor information data collection by cluster head. Second, finding a path towards the base station using ants in the cluster head, helping in determining the next hop. In the third step, ants that passed by the base station collect path information. Moreover, each cluster head can find the best cluster head candidate to forward its packets. Lastly, the transmission process starts forwarding the data packet. The proposed approach was compared with other studies in the literature, showing that the proposed work outperformed in terms of routing efficiency and network lifetime.

In order to enhance the data aggregation efficiency in a multi-hop wireless sensor network, Sert et al. [46] introduced a two-tier distributed fuzzy logic-based protocol (TTDFP). In tier I, TTDFP selects the optimum cluster head through an energy-based competition of provisional leaders using a probabilistic model. In all phases, the inclusion of a central decision-making point does not require in TTDFP. In the fuzzy clustering phase, TTDFP handles specific events in the clustering phenomena more efficiently compared with its crisp and other fuzzy counterparts. Moreover, TTDFP utilizes the optimization framework to tune the two parameters in this tier, which are the threshold and the maximum competition radius, rather than the use of a trial-and-error approach to find the right blend of these parameters. 
On the other hand, in the second tier (tier II), fuzziness enhances routing performance in comparison with its crisp counterpart. In mission-critical sensitive scenarios, TTDFP is an efficient approach to be used, where utilization context may be updated concerning the application field. In the clustering phase, TTDFP uses three main inputs: the remaining node energy, distance, and relative node connectivity. In the routing phase, TTDFP increases the operation architecture of multi-hop routing methodology with the use of fuzzy logic. TTDFP employs two fuzzy parameters, namely, distance to the efficient routing path and residual energy. In order to evaluate the performance of the proposed protocol, several experiments were conducted, proving that TTDFP is an efficient protocol compared with other related work proposed in the literature.

One big challenge in a wireless sensor node is that it has very little storage space, low computational capability, and limited battery power. Due to the aforementioned issues, a trade-off should be handled between power optimization and processing accuracy in WSNs. In a study by Tabatabaei et al. [47], the authors proposed a novel energy-aware clustering method via algorithm (LPO) and fuzzy logic in WSNs. Figure 7 depicts a clustering technique, which uses the FLS described in Figure 1, that has been provided based on two parameters named distance from the sink and remaining energy. The proposed clustering technique has a direct effect on the routing process as it takes the nodes' distances from the sink while forming the clusters, which results in a superb performance in terms of end-to-end delay, energy consumption, input packet, and network lifetime when compared with the related literature work.

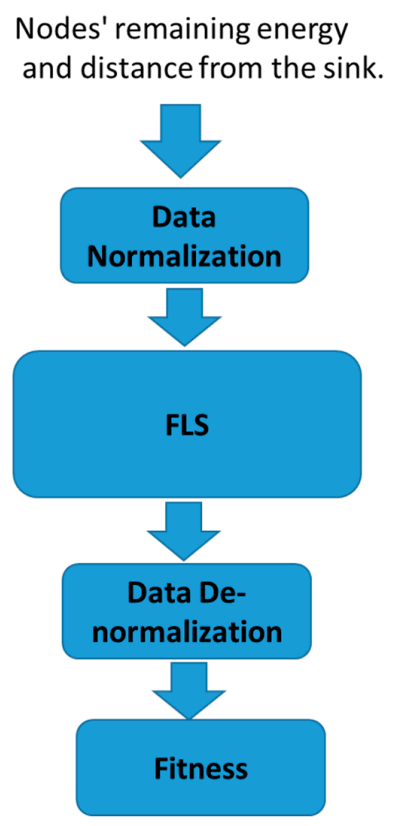

Figure 7. Forwarding mode fuzzy monitoring system.

Typically, data are received by sensor nodes within a limited transmission range. These data have been extracted from the packet and processed then forwarded to the sink node. This process leads to high-energy consumption. This issue is considered one of the main challenges in WSNs that have a direct impact on the lifetime as sensors use batteries. Moreover, sensors that are closer to the sink consume higher energy than others because they directly forward the packets to the sink. Therefore, having an adaptive energy transmission based on the nodes' positions compared to the sink node is considered as one of the major issues in WSNs.

In [48], Koosheshi and Ebadi proposed optimization energy consumption with multiple mobile sink nodes using fuzzy logic. In this paper, a technique is suggested that can optimize the energy consumption of sensor nodes through various mobile stations (MSs) in which fuzzy logic is used in WSNs. In this technique, instead of using a fixed sink at a predetermined location, an MS is used to 
collect sensor information so that it moves smartly within the network and collects sensor information. The remainder of the nodes are also grouped on the basis of fuzzy logic in order to avoid the issue of the energy hole. The fuzzy logic concept is centered on the idea of partial set membership, rather than crisp or discreet set membership. It was initially implemented as an alternative strategy to information processing that is defined by a set of "fuzzy parameters" that include components in which membership level varies within the set. Fuzzy logic differs from standard control techniques by incorporating simple IF-THEN structure instead of any complicated mathematical model.

Consequently, the lifetime of the network is therefore improved. The purpose behind this study, however, is to solve the issue of the energy hole, particularly in large networks, by separating the WSN field into different independent zones and using an MS in each. They also use the fuzzy logic method in this study to deal with challenges in the estimation of cluster head radius. In the purposeful fuzzy strategy in this algorithm, the selection of $\mathrm{CH}$ happens by selecting three fuzzy descriptors, namely, residual energy of each node, distance to $\mathrm{RN}$, and node density in order to estimate the radius of competition for tentative $\mathrm{CHs}$.

Indeed, the introduced multiple mobile sink nodes had a positive impact on the routing process, as it made the destination node(s) closer to the sensor nodes, thus reducing the needed transmission energy and improving the network lifetime. In this algorithm, multi-sink nodes' MSs are employed, and the positive area defined as the areas that have neighbors or eligible nodes are divided into small areas. Moreover, the distances between sensors are reduced by proposing a clustering method using fuzzy logic. This method helps in reducing energy consumption. First node die (FND) and half of the nodes alive (HNA) metrics have been tested. Simulation results outperformed other algorithms in terms of energy consumption. As a result, the problem of the energy hole in WSNs was optimized.

WSN suffers from computation power, limited energy, bandwidth, and resource memory. The use of clustering techniques in the routing protocol is one promising solution that has a direct impact on energy efficiency in WSNs. In [49], Balaji et al. proposed a hop-by-hop protocol named the development of a fuzzy-based energy-efficient cluster routing protocol to increase the WSN lifetime. The algorithm has several phases. In the setup phase, clustering techniques in routing protocols are used to identify and select the $\mathrm{CH}$. Next, the data are forwarded from sender $\mathrm{CH}$ to another $\mathrm{CH}$ towards the sink node. In the second phase, the proposed protocol uses fuzzy logic with three parameters that were weight, energy, and distance, to forward the data from the source node towards the sink. The use of the fuzzy logic helps in identifying the node that has the highest trust factor and closer to the sink, thus improving the routing process. Then, $\mathrm{CHs}$ are selected on the basis of these factors. Simulation results showed that the proposed protocol improved the network lifetime and reduced the network overhead; therefore, the network lifetime and overhead were improved.

To sum up, comparison tables for the surveyed papers that highlight the most common routing protocols/techniques in WSNs that use fuzzy logic algorithms are presented in Table 3. In this table, the comparison between various fuzzy logic routing protocols in WSNs is summarized. As shown in this table, energy consumption is considered to be the main problem in routing algorithms. Each sensor node is equipped with batteries with limited energy. The energy of each sensor node should be balanced in order to improve the network lifetime. Most algorithms in the literature focus on energy-efficiency with different modeling parameters. Residual energy has been used as main input for the fuzzy system. Other parameters have been utilized such as link quality, distance, and number of hops, and some algorithms have utilized cluster head selection in order to balance the energy and avoid the void region. Different fuzzy logic strategies have been utilized by the survey algorithms. FEARM [43] utilized different parameters such as link quality, energy consumption, physical distance, and available buffer, whereas FLGR [44] did not employ physical distance parameter. On the other hand, new and colony optimization routing [45], TTDFP [46], and LPO [47] focused on identifying cluster head based on different parameters. The main forwarding strategies in most algorithms have been designed to select the efficient sensor node that is closer to the sink node with minimum route cost. 
Table 3. Main features of fuzzy-based WSN routing protocols.

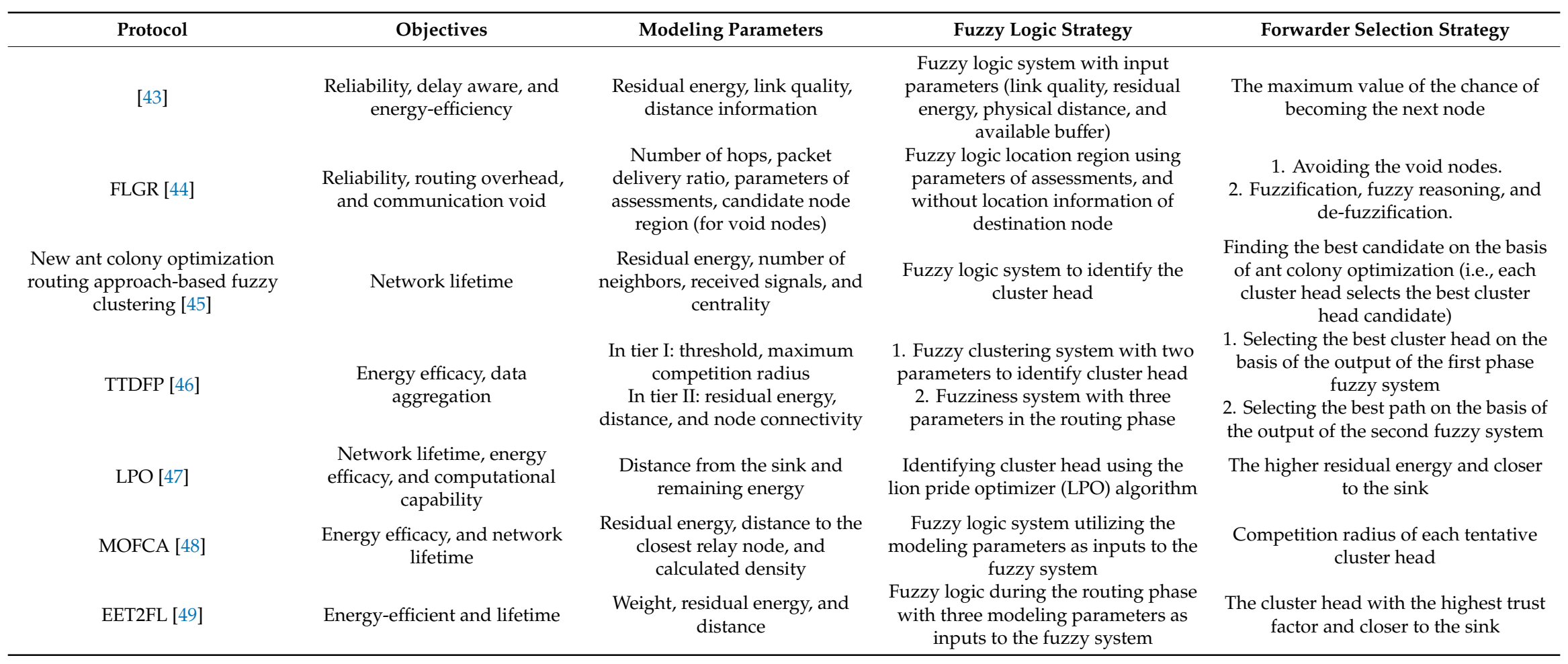




\section{Fuzzy Logic-Based WSN Power Management and Optimization}

The third essential and determining factor that affects the network lifetime is the power management and optimization technique used in turning ON/OFF the sensor nodes, thus prolonging their batteries and extending the network lifetime. Figure 8 depicts the different phases that a sensor node goes through. In a normal situation, it should operate in a sleep mode, where it consumes the minimum amount of energy by shutting down its main functioning peripherals, sensors, and communication modules. These nodes will wake up after a specific time that is normally specified by the application. In this phase, the senor node awakes its peripherals and sensors to capture the data of interest, and then either go back to the sleep phase or transmit the captured data by waking up the communication modules. In what follows, a comprehensive literature review for the recent and most prominent works that utilize fuzzy logic in sensor nodes' power management is presented.

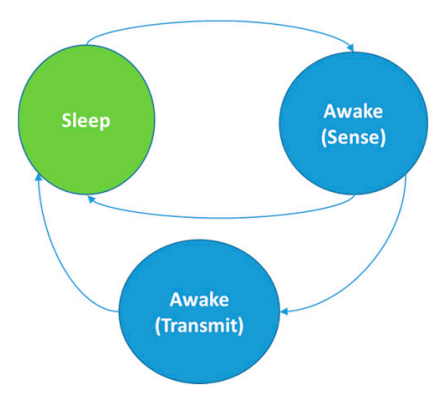

Figure 8. Sensor node different energy-saving modes.

Colletta et al. [50] focused on the IEEE 802.15.4-based industrial WSN protocol and proposed a new mechanism to control the uptime of the nodes using a fuzzy logic algorithm. WSNs in industry communications undertake high reliability and low power consumption. The basic function of industrial WSN is to generate a highly reliable and self-healing industrial system. Therefore, in order to increase the lifetime of the nodes and network, the authors proposed a new energy management mechanism that is capable of prolonging the network lifetime through a sleep/wakeup policy using a fuzzy logic algorithm. The objective of the mechanism was to build a time-driven approach that woke up the nodes only when they were needed by the application under consideration. The goal of the proposed mechanism was to show how to use a fuzzy logic controller in industrial WSN, and how to increase the lifetime of the batteries of the individual nodes by continuously controlling the individual nodes' energy consumption. The proposed mechanism studies the correlation between the sampled data, and if a high correlation is found, the sampling period is decreased, thus increasing the sleeping time and saving energy. The proposed algorithm called dynamic sampling algorithm (DSA) utilizes four input variables coming from the sensors, namely, the deadline miss ratio, deadline miss ratio desired, old sampling time, and remaining battery capacity, which help the network to manage the energy resources of its nodes. The main goal of DSA is to awaken the nodes only when it is needed for the application that manages and controls their operation. DSA is built on the basis of a fuzzy logic controller, it is linked with the sink node, and in case of large WSN, it is linked with router nodes. Figure 9 depicts the fuzzy controller in the DSA approach, which uses the FLS described in Figure 1. Depicted in Figure 9 are the deadline miss ratio (E), deadline miss ratio desired (DE), remaining battery capacity ( $R B C)$, and old sampling time $(\mathrm{T})$ variables, which are the inputs to the controller as analog-digital shape. The variables are then converted to a "linguistic" shape on the basis of the membership functions that may be enhanced at the design time. The linguistic values are positive big, positive small, zero, negative small, and negative big. 


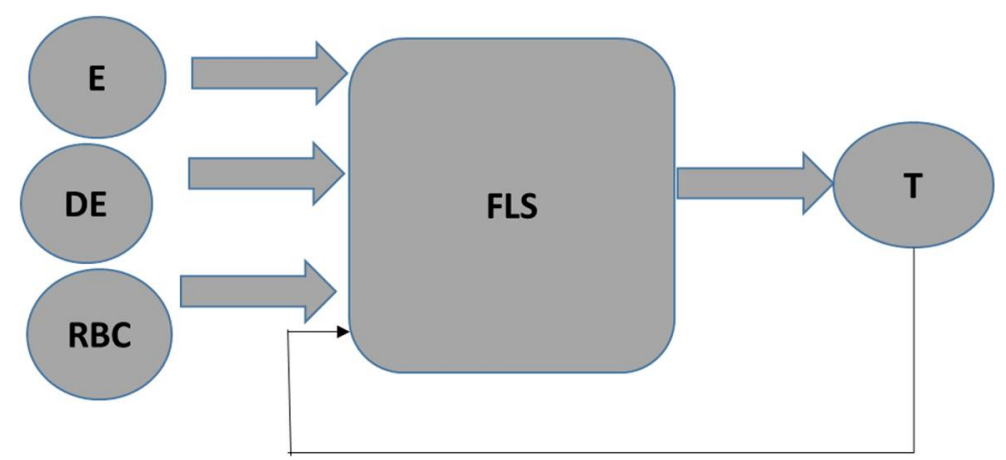

Figure 9. The fuzzy controller in dynamic sampling algorithm approach. E: deadline miss ratio, DE: deadline miss ratio desired, RBC: remaining battery capacity.

These linguistic values are processed using an inference mechanism on the basis of a set of inference rules using a series of IF-THEN statements, which determine the output linguistic values on the basis of the input variables. The output linguistic values need to be transferred by a controller to analog values readable by the sensors. This approach allows the controller to use more input information about the network status. This information gives good accuracy, and therefore yields superior performance in terms of energy-saving and WSN life cycle.

Another work presented by Colletta et al. [51] applied a fuzzy logic algorithm by optimizing the sleep/wakeup process in the most common industrial communication protocols for WSNs, namely, wireless highway addressable remote transducer(HART) and IEEE 802.15.4 protocols. The authors proposed an innovative management algorithm of energy resources in order to minimize the average power consumption of each slave node, by activating the nodes only in case of critical events, thus prolonging the network lifetime. This algorithm consists of a fuzzy logic algorithm that can manage sleep/wakeup messages sent by the network controller to their slaves dynamically on the basis of the dynamic power management algorithm (DPMA). The DPMA algorithm wakes up the nodes only when they are needed, and the input variables are the sampled data and the sampling time to the fuzzification block of its fuzzy controller when the fuzzy logic controller is activated. The controller operates two input variables: the current sampling time and the data of interest (the detected temperature value, for example), where it utilizes a sampling time membership function and the detected data, and then passing the output to the de-fuzzification block, with the output being a new sampling time that is produced and used in the sampling process and wakeup/sleep cycle. Figure 10 depicts the used fuzzy controller scheme of the proposed system.

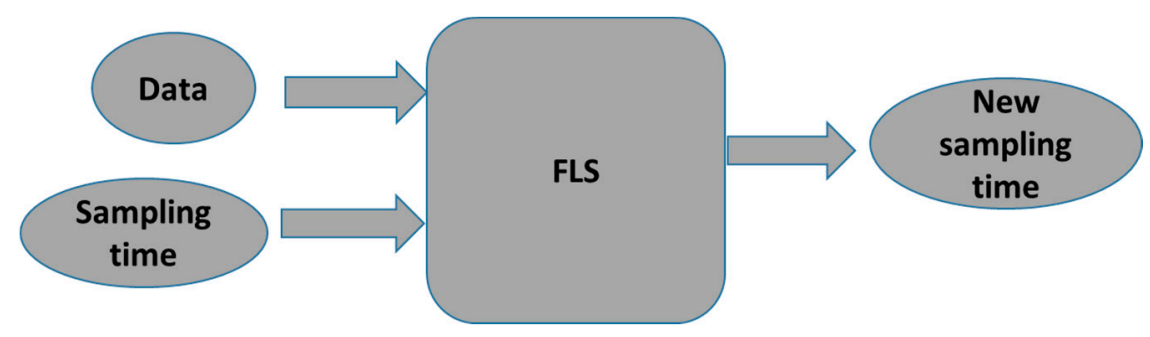

Figure 10. Fuzzy controller scheme.

The algorithm uses the temperature as input data with the range of -40 to $75^{\circ} \mathrm{C}$, and the current sampling time produces a new sampling time on the basis of a fuzzy controller, as depicted in Figure 10, which uses the FLS described in Figure 1. The fuzzy system evaluates the temperature variations using a specific inference mechanism, and the sampling time is a value varying from 0.1 to 1 seconds. The input variables are set in numerical shape. Every input variable has three Mamdani membership functions (low, medium, and high), then the values are processed by the fuzzy controller using an 
inference mechanism utilizing a set of IF-THEN statements. The new sampling time, represented by a membership function, will be then fuzzified through the centroid algorithm and converted to a numerical value readable by the sensors.

Pau. et al. [52] proposed a power consumption reduction mechanism using fuzzy logic that utilizes the battery level and ratio throughput for identifying the sensor sleep time on the basis of the IEEE 802.15.4 protocol. Figure 11 depicts the proposed fuzzy logic controller. It calculates the sleep time on an IEEE 802.15.4 network using a centralized mechanism. The aim of sleep time calculation is to prolong the WSN lifetime for monitoring purposes. The fuzzy logic controller is used to calculate sleep time on reduced function device (RFD). After that, RFD will notify the first pan coordinator (FPC) when the sleep time is finished. The proposed mechanism uses three membership values, namely, sleeping time is the output variable, whereas the ratio of throughput to workload (TH/WL) and battery level are the input variables.

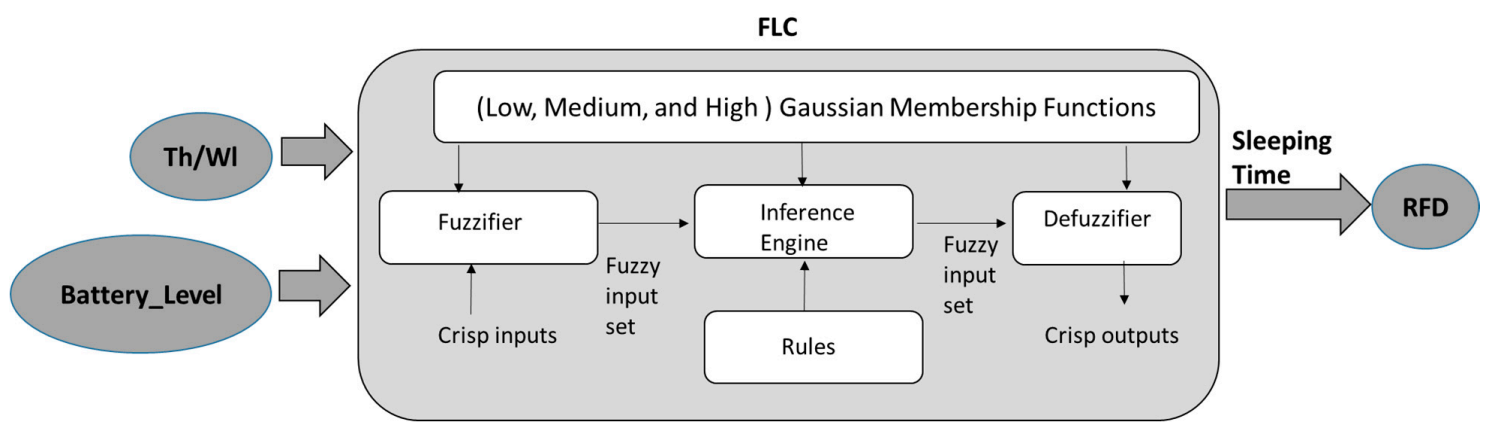

Figure 11. The proposed FLC architecture. TH/WL: throughput to workload.

The FLC defines the sleeping time of the RFD on the basis of the battery-level and the ratio of throughput to workload. The throughput is the aggregate for periodic and aperiodic packets sent by each node. The workload is the total number of packets that each node has sent. Each input and output variable has three Mamdani membership functions (low, medium, and high). These functions fuzzify the crisp inputs, and the range of throughput-to-workload percentage is 0 to $100 \%$, with the battery level being 0 to 1024 . The output variable has a sleeping time with a range from 0 to $10 \mathrm{~s}$. The sampling time value is a constant determined at the design time for each node. Gaussian fuzzy membership functions are used for input and output variables, where the degree of membership is represented by normalized values between 0 and 1 . The output value is determined using fuzzy rules according to the IF-THEN statement. The FLC converts fuzzy output variables into crisp values by using the de-fuzzification process, so that the values of the variable are retable by the sensors. The de-fuzzification method used in this study is the centroid of area method, which is evaluated for each membership function for each rule. The output is then determined as the average of the individual centroid weighted by their membership values.

Masdari et al. [53] proposed a distributed fuzzy logic-based sink selection mechanism for one-hop WSN that manages the nodes' selection process to the sink nodes. Having multiple sink nodes in WSNs can improve network throughput and reduce packet loss and energy consumption. In this study, a fuzzy logic-based algorithm was used to assign a sink node for each node on the basis of the network congestion status between the sink and ordinary nodes. This one-hop network paradigm not only reduces the transmission delay but also reduces the packet loss due to the high congestion encountered in single sink-multiple hop network architecture. The input variables of the fuzzy logic controller are congestion, energy, and distance between a node and the sink. Figure 12 depicts the proposed fuzzy sink selection, which uses the FLS described in Figure 1. This algorithm was designed to avoid congestion at the sink nodes and distribute the nodes' loads between multiple sink nodes. Each node uses a fuzzy inference engine, which receives information such as distance to sink, the energy of node, and the average load on each sink. By using the proposed algorithm, each node can choose the closest 
and uncongested sink to transfer its data to it. The results obtained from simulations indicated that the proposed mechanism can minimize the congestion in the sink nodes and balance the nodes' load on multiple sink nodes. Moreover, the proposed mechanism can minimize the transmission power consumption and delay by minimizing the number of packet retransmissions to the sink nodes.

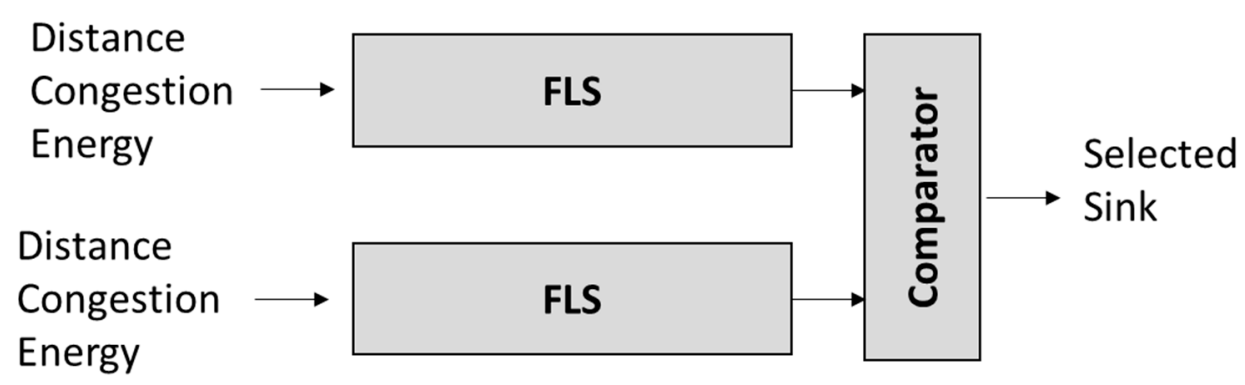

Figure 12. The proposed fuzzy sink selection paradigm.

Furthermore, the authors proposed a distributed fuzzy sink selection solution consisting of the following: a fuzzification module, a knowledge base, an inference engine, and de-fuzzification module. The input linguistic variable distance is used to represent the distance between a node and its sink. This variable has three Mamdani membership functions: close, mid, and far, whereas the input linguistic variable congestion is used to indicate the average congestion of a sink node in previous rounds. This variable has three Mamdani membership functions: low, medium, and high. Finally, the input linguistic variable energy is used to represent the remaining energy of nodes. This variable has three Mamdani membership functions: low, medium and high. As the fuzzification step is performed, the gained membership values are applied to the IF-THEN rules to define the fuzzy output set. The authors used the Mamdani method as a fuzzy inference method. In the de-fuzzification step, the crisp value represents the suitability of the sink node and the sensor node selects the sink, which has the highest value of the sink output variable. In this scheme, the COA is used in the centroid de-fuzzification.

The authors in [54] proposed a fuzzy logic-based mechanism utilizing the particle swarm optimization (PSO) algorithm that works with the challenging nature of the energy consumption in industrial wireless sensor network (IWSN). The proposed mechanism is an extension of the work done in [52]. The input variables of the fuzzy system are the battery level and the ratio of throughput to workload, which define the sleeping time of sensor devices in an IWSN on the basis of the IEEE 802.15.4 protocol. The authors added PSO to get the best values concerning the battery life of nodes and optimizing the membership functions by changing their range parameters. The results of the proposed mechanism achieved a real power consumption reduction and prolonged the network lifetime. Further, the algorithm used three input variables: sleeping time, battery level, and the ratio of throughput to workload. Moreover, the proposed FLC employs three membership functions (low, medium, and high) for each input and output linguistic variables. The FLC must convert its fuzzy output variables in crisp values by using de-fuzzification method so that the system can understand them. Several methods can accomplish the de-fuzzification process. In this study, the COA method was selected. In this scheme, the COA of each membership function, for each rule, is first estimated. The final output is then measured as the average of the individual COA weighted by their membership values.

Cao et al. [55] proposed a fuzzy logic-based adaptive energy management mechanism through the particle swarm optimization algorithm (FLC-PSO) in order to improve the energy efficiency for WSN. The proposed mechanism considered the most important performance factors of WSN, namely, the overall remaining energy and the number of dead nodes, which were used as the input variables of the fuzzy system depicted in Figure 13. The aim of using PSO is to optimize the membership functions, and the optimization goal is to minimize the number of dead nodes and maximize the remaining energy in order to reach an optimal fuzzy logic controller. Simulation results proved that the proposed FLC-PSO 
mechanism outperformed its literature counterparts. Therefore, FLC-PSO provides an efficient energy management mechanism for WSN.

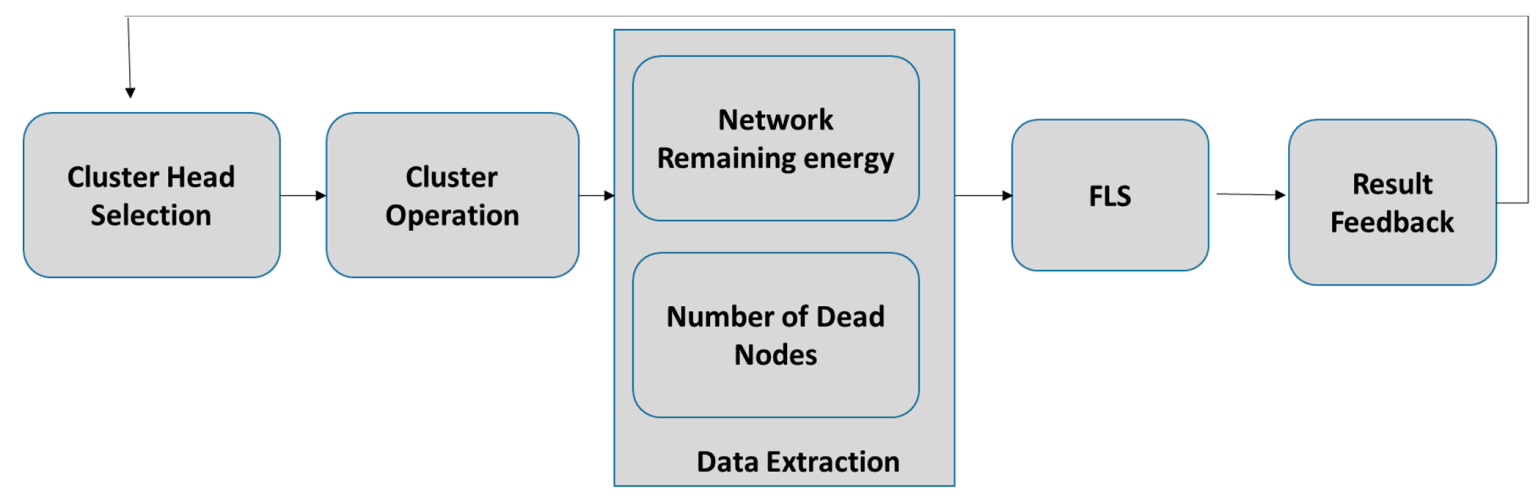

Figure 13. The FLC model.

Pau et al. [56] proposed a fuzzy logic-based algorithm to overcome the WSN energy consumption problem in battery device nodes in an IEEE 802.15.4 network for smart home applications. Considering the duty cycle, the sleeping time of nodes is dynamically set by the FLC. The proposed mechanism in this work is an extension of the work presented in [54]. The main goal of this work was to design a system that could be executed using off-the-shelf hardware. In this study, a different type of membership function was used in the FLC to realize a practical method that reached the goal while avoiding complicated and computationally costly solutions, such as PSO, which would decrease the possible applicability of the procedure in real situations. The goal was to develop a proof-of-principle implementation utilizing off-the-shelf devices, highlighting that the suggested method does not demand compelling hardware and can be openly realized on low-cost devices. Similar to the work presented in [54], the proposed mechanism used two input variables to the FLC: throughput to workload and the device battery level. Further, it had three membership functions: throughput to workload, the device battery level, and sleeping time.

The proposed method operates as follows: the first pan coordinator node utilizes an FLC to realize the output values of the sleeping_time of each reduced function device. The FLC fixes the sleeping_time of the RFD by considering the ratio of throughput to workload and the battery_level. The FLC has three membership functions (low, medium, and high) for the input variables. These functions fuzzify the crisp inputs, and the range for input variables are 0 to $100 \%$, with 0 to 1024 for the TH/WL and battery_level, respectively. Furthermore, the output variable has three membership functions (low, medium, and high); the range of the crisp values of this output variable is 0 to 10 s. Trapezoidal membership functions are applied to the TH/WL, battery_level, and sleeping_time, where the degree of the membership is represented by normalized values from 0 to 1 , and the output value is obtained on the basis of nine IF-THEN fuzzy rules. The FLC converts internal fuzzy output variables into crisp values through the de-fuzzification method so that the system can understand these variables. The de-fuzzification used in this study is the COA method.

Moreover, as in most of fuzzy-based power management algorithms for WSNs discussed in the literature, the metrics such as network lifetime and battery level were used to evaluate the proposed algorithms in [50-56]. The authors validated the performance of the proposed technique under different network densities. Table 4 depicts the network lifetime improvement percentages calculated as follows: 
Table 4. Lifetime improvement percentages.

\begin{tabular}{cc}
\hline Fuzzy-Based Power Management Algorithms for WSNs & Network Lifetime Improvement (\%) \\
\hline DSA [50] & $37.5 \%$ \\
DPMA) [51] & $14.0625 \%$ \\
Power consumption reduction mechanism using fuzzy logic [52] & $30.76 \%$ \\
Distributed fuzzy logic-based sink selection algorithm [53] & $20 \%$ \\
Fuzzy logic-based mechanism utilizing particle swarm & $54.545 \%$ \\
optimization algorithm [54] & $6.666 \%$ \\
FLC-PSO [55] & $41.666 \%$ \\
\hline
\end{tabular}

Network lifetime improvement $(\%)=[($ new lifetime - original lifetime $) /$ original lifetime $] \times 100 \%$ Finally, Table 5 summarizes the surveyed papers related to the most important power management and optimization mechanisms in WSNs that utilize fuzzy logic. 
Table 5. The main features of the surveyed fuzzy-based power management algorithms for WSNs.

\begin{tabular}{|c|c|c|c|c|}
\hline Mechanism & Objectives & Modeling Parameters & Fuzzy Logic Input Variables & $\begin{array}{c}\text { Minimize Power } \\
\text { Consumption Strategy }\end{array}$ \\
\hline DSA [50] & $\begin{array}{l}\text { Increase lifetime of the individual } \\
\text { nodes batteries }\end{array}$ & $\begin{array}{l}\text { Deadline miss ratio, deadline } \\
\text { miss ratio desired and } \\
\text { remaining battery capacity }\end{array}$ & $\begin{array}{c}\text { Fuzzy logic system with input } \\
\text { parameters (deadline miss ratio, } \\
\text { deadline miss ratio desired, old } \\
\text { sampling time, and remaining battery } \\
\text { capacity) }\end{array}$ & $\begin{array}{c}\text { Timing of sleep and wakeup } \\
\text { policy }\end{array}$ \\
\hline DPMA [51] & Increases the whole network lifecycle & $\begin{array}{l}\text { Current sampling time and the } \\
\text { temperature value detected }\end{array}$ & $\begin{array}{l}\text { Fuzzy logic system evaluates the } \\
\text { temperature variations. Input } \\
\text { parameters: sampling time and data. }\end{array}$ & $\begin{array}{l}\text { Optimization of the } \\
\text { sleep/wakeup mechanism }\end{array}$ \\
\hline $\begin{array}{l}\text { Power consumption reduction } \\
\text { mechanism using fuzzy logic [52] }\end{array}$ & Improves the lifetime of WSN nodes & $\begin{array}{l}\text { Sleeping time, battery level, and } \\
\text { ratio of throughput }\end{array}$ & $\begin{array}{l}\text { Fuzzy logic controller to calculate } \\
\text { sleeping time according to the battery } \\
\text { level and throughput-to-workload }\end{array}$ & $\begin{array}{l}\text { Dynamically changes the } \\
\text { sleeping time to increase the } \\
\text { battery life }\end{array}$ \\
\hline $\begin{array}{l}\text { Distributed fuzzy logic-based sink } \\
\text { selection algorithm [53] }\end{array}$ & $\begin{array}{c}\text { Reduces the delay and improves the } \\
\text { lifetime of WSN }\end{array}$ & $\begin{array}{l}\text { Distance, energy, and } \\
\text { congestion }\end{array}$ & $\begin{array}{c}\text { Fuzzy logic controller inputs are } \\
\text { neighbor nodes number, remaining } \\
\text { battery energy, and distance between } \\
\text { a node and a sink }\end{array}$ & $\begin{array}{l}\text { Minimizing the number of } \\
\text { packet retransmissions delivery } \\
\text { to the sink by selecting the } \\
\text { proper sink per node }\end{array}$ \\
\hline $\begin{array}{l}\text { Fuzzy logic-based mechanism } \\
\text { utilizing particle swarm } \\
\text { optimization algorithm [54] }\end{array}$ & $\begin{array}{l}\text { Power consumption reduction and } \\
\text { prolonging the lifetime of node and } \\
\text { network in industrial wireless sensor } \\
\text { networks }\end{array}$ & $\begin{array}{l}\text { Sleeping time, battery level, and } \\
\text { the ratio of throughput }\end{array}$ & $\begin{array}{l}\text { Fuzzy controller input variables are } \\
\text { sleeping time, battery level, and the } \\
\text { ratio of throughput }\end{array}$ & $\begin{array}{l}\text { Optimizing the member } \\
\text { functions (MFs) by varying their } \\
\text { range }\end{array}$ \\
\hline FLC-PSO [55] & $\begin{array}{l}\text { Minimize the number of dead nodes } \\
\text { and maximize the remaining energy }\end{array}$ & $\begin{array}{l}\text { Remaining energy and the } \\
\text { number of dead nodes }\end{array}$ & $\begin{array}{l}\text { Fuzzy logic input variables are } \\
\text { remaining energy and the number of } \\
\text { dead nodes }\end{array}$ & $\begin{array}{l}\text { Optimizing membership } \\
\text { functions and FLC }\end{array}$ \\
\hline $\begin{array}{l}\text { A fuzzy logic-based algorithm for } \\
\text { smart homes [56] }\end{array}$ & $\begin{array}{l}\text { Overcome energy consumption in } \\
\text { battery nodes in an IEEE } 802.15 .4 \\
\text { network for smart homes and design a } \\
\text { system that can be executed using } \\
\text { off-the-shelf hardware }\end{array}$ & $\begin{array}{l}\text { Throughput to workload, the } \\
\text { battery level of the device, and } \\
\text { sleeping time }\end{array}$ & $\begin{array}{l}\text { Fuzzy logic controller with } \\
\text { throughput-to-workload and the } \\
\text { node battery }\end{array}$ & $\begin{array}{c}\text { Dynamically sets the sleeping } \\
\text { time to increase the battery } \\
\text { duration }\end{array}$ \\
\hline
\end{tabular}




\section{CMR-WSN Mobility Management Utilizing Fuzzy Logic}

Mobility is one of the main advantages of CMR-WSN over classical WSN, as mobile nodes (robots in this case) can move within the AoI to perform extended sensing mechanisms for areas not reachable by static sensors. However, mobility management protocol is required to maintain the connectivity of the mobile nodes (the robots in this case) during the nodes' movement. Therefore, a proper mobility management protocol must consider all the necessary actions to support the movement of mobile users without losing connectivity. The major process of a mobility management protocol is called handoff. The handoff process involves three phases: (a) triggering, (b) decision, and (c) execution. In the literature, several handoff-triggering techniques have been proposed. Most of them rely on the following criteria:

- Received signal strength indicator (RSSI) threshold - when the RSSI is below a predefined value, then the handoff is triggered.

- Link loss-if the link loss of the mobile node is above a predefined value, then the handoff is initiated.

- RSSI moving average - if the average value of the RSSI over a period is below than a predefined value, then the handoff is triggered.

- Exponential weighted moving average-if the moving average of the RSSI over a period is below a predefined value, then the handoff is triggered.

Link loss denotes the ability of the mobile node to successfully communicate directly with the parent node while also considering any retransmissions at the Medium Access Control (MAC) layer. Figure 14 shows the behavior of RSSI during a random walk in an industrial environment and, more specifically, in a refinery [57]. It is obvious that RSSI is used as a single parameter to decide whether the handoff triggering will produce unnecessary overhead in terms of energy consumption and computation due to the scanning period for searching new attachment points, and this is due to its unpredictable behavior. On the other hand, using the link quality as a metric to initiate the handoff poses another drawback, which is the number of packets needed to achieve reasonable accuracy. Averaging techniques such as moving average and exponential weighted moving average can provide solutions to some of the drawbacks of using a single value of RSSI or link loss.

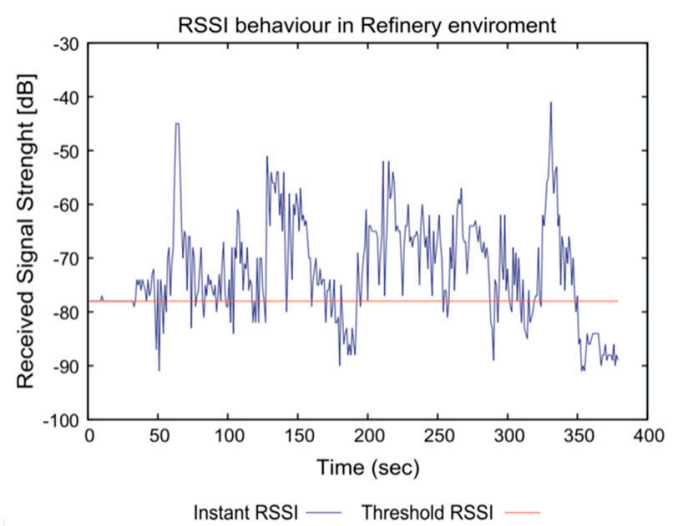

Figure 14. Received signal strength indicator (RSSI) behavior in a refinery environment [57].

Single metrics, such as RSSI, are not suitable to trigger the handoff procedure, especially when considering wireless sensor networks in dynamic environments [58-61]. To solve these performance issues (increased energy consumption and a large number of unnecessary triggers) introduced by the usage of solely metrics such as RSSI and link loss, researchers started investigating different techniques to control the handoff procedure. One such technique is to use fuzzy logic. The reason for using fuzzy logic resides on the fact that: 
- It can control nonlinear systems based on observable phenomena.

- It is flexible due to the option to easily modify the expert-defined rules and tune the membership functions so that it can achieve the desired performance.

- It can work without training and learning as other solutions such as neural network do. A fuzzy-logic system can be built on the basis of the experience of people who already understand the system.

In what follows, the most related fuzzy logic-based mobility management mechanisms proposed in the literature are summarized. In [62], the authors introduced MoMoRo, a mobility layer that can be easily integrated into existing systems enabling the mobility of the nodes. MoMoRo uses a fuzzy estimator to make link quality estimations and detect route disconnections. MoMoRo was evaluated both in indoor and outdoor testbeds. The triggering of the handoff is initiated by the link packet losses, and the selection of the new attachment point is based on the fuzzy logic estimator.

In [63], the authors proposed an effective mechanism to guarantee the performance of handoff, including a mobility-aware scheme, temporary connection, and quick registration. The main contribution of this paper is that the proposed mechanism is implemented not only in the proposed testbed but also in a real industrial environment. The results indicated that the proposed mechanism not only improved the accuracy of handoff triggering but also solved the problem of the ping-pong effect during handoff. The authors of [64] presented a fuzzy rule-based simulation system for WSN to predict the node's mobility and energy. Mobility prediction provides a stable path for a network and energy prediction increases the lifetime of the network. Network simulator NS3 is used for simulation results. In this paper, the handoff decision for heterogeneous networks was identified as a fuzzy multiple attribute decision-making problem, and fuzzy logic was applied to deal with the imprecise information. In [65], a handover algorithm was proposed to support vertical handovers between heterogeneous networks. This was achieved by incorporating the mobile IP principles in combination with fuzzy logic concepts utilizing different handover parameters. Furthermore, in [66], the authors deal with a vertical handover decision algorithm based on the fuzzy control theory. The algorithm takes into consideration the factors of power level, cost, and bandwidth in order to decide about the vertical handover. In [67], the authors proposed and implemented a fuzzy-based handover system (FBHS), where they showed that the proposed system had good behavior for handover enforcement, but in some cases, it could not avoid the ping-pong effect.

In [68], the authors proposed a fuzzy logic mobility controller (FLMC) in order to support critical applications such as personnel safety and healthcare monitoring. The main idea was to use a combination of two existing metrics that are locally available at each node with the purpose of predicting end-to-end losses and supporting the triggering of the handoff procedure. FLMC was evaluated in a real environment and, more specifically, in a refinery area. As mentioned previously, FLMC operation relies on two locally available metrics, the RSSI and the link loss. As a first step, several experiments were run in the refinery area in order to investigate the behavior of the two metrics and extract information about the relationship of RSSI, link loss, and end-to-end packet loss. Figures 15 and 16 show the relationship between the aforementioned metrics, and the FLMC controller with the inputs and outputs, respectively. More specifically, on the basis of Figure 15, we can conclude the following:

1. For link loss above $15 \%$ and RSSI less than $-78 \mathrm{dBm}$, the end-to-end packet loss was significant and clearly not acceptable.

2. For RSSI greater than $-60 \mathrm{dBm}$ and the link loss up to $40 \%$, the end-to-end packet loss was acceptable. This happened because the mobile was not able to retransmit the lost packets

In the proposed fuzzy controller, as shown in Figure 16, the quantities were considered at the discrete instant $\mathrm{kT}$ :

3. T denotes the sampling period. 
4. RSSI (kT) is the received signal strength taken every $\mathrm{kT}$.

5. LL (kT) denotes the rate of link loss measured at kT.

6. $\mathrm{Pd}(\mathrm{kT})$ is the calculated decision probability. This probability is taken into account to trigger the handoff procedure.

7. $\mathrm{SGi}_{1,2}(\mathrm{kT})$ is the input scaling gains.

8. PThreshold is a predefined value that is compared to the $\operatorname{Pd}(\mathrm{kT})$ and denotes if the specific $\operatorname{Pd}(\mathrm{kT})$ will trigger the handoff or not.

In order to further improve the performance of the FLMC solution, the authors implemented an adaptive thresholding solution [69] with the main target to reduce the power consumption and to increase the on-time triggering. Furthermore, in [70], the authors extend their solution to support adaptive fuzzy logic operation to produce a successful handoff. The evaluation results clearly show that the adaptive fuzzy logic mobility controller outperformed the non-adaptive fuzzy logic controller in terms of all evaluation metrics (packet loss, power consumption, and on-time triggering).

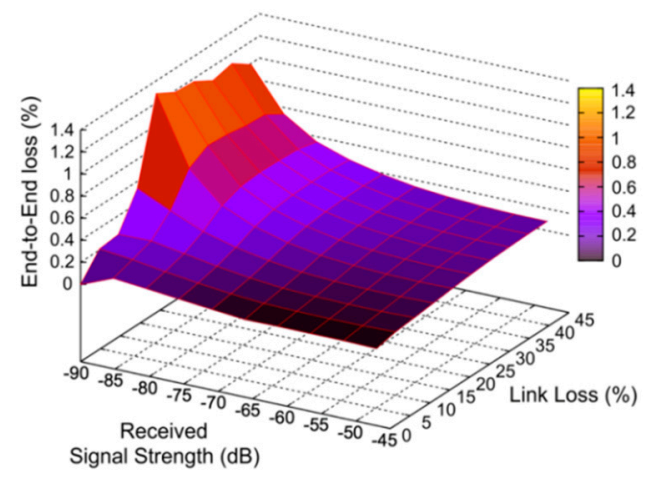

Figure 15. Relationship between RSSI, link loss, and end-to-end loss [57].

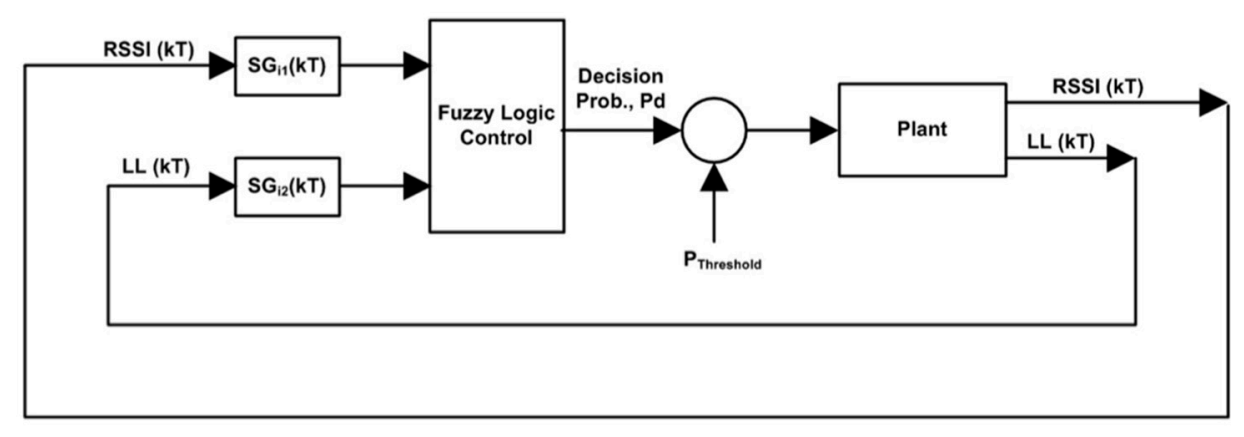

Figure 16. Fuzzy logic-based mobility controller [57].

Table 6 depicts the latest proposed solutions to support mobility in wireless sensor networks. The table summarizes the objectives of each protocol, the metrics used as input to the fuzzy logic controller, and, finally, the evaluation environment. The two out of four protocols were evaluated in a real industrial environments $[57,63]$ and under harsh conditions, which makes them applicable for unpredictable CMRS environments. The FLMC [57] had the least overhead among all the fuzzy-based mobility management solutions and used only two metrics (RSSI, link loss) that were locally available to all nodes. Processing only two available metrics will help with the design of an optimal controller that can optimize the performance of the system. There are many challenges for cooperative multi-robotic wireless sensor network systems, especially when the mobility of the robots is considered. The mobility management solutions provided should be intelligent enough to improve the overall performance of the system. Therefore, any proposed mobility protocol should provide controlled performance considering the following main characteristics of mobile robots: 
1. High number of mobile robot systems with embedded wireless sensors.

2. Dynamic topology.

3. Limited resources (e.g., power, computation).

All of these characteristics must be addressed in order to achieve a controlled system that supports an efficient and effective communication protocol between mobile robots. In addition, on the basis of the evaluation presented in the aforementioned papers, we can only compare the results from three of them $[57,63,69]$ in terms of packet losses, number of handoffs, and power consumption. Due to the different evaluation parameters and testbed field, it is not possible to directly compare the solutions; therefore, we decided to compare the performance of the proposed solutions with the RSSI-based solution.

Figure 17 shows the comparison of ireless controller area network (Wireless CAN) [63], FLMC [57], and adaptive-FLMC [69] in terms of packet losses. It is obvious that all the proposed solutions decrease the packet losses compared to the RSSI-based solution. More specifically, Wireless CAN reduces packet loss by $73.8 \%$, FLMC by $69.8 \%$, and adaptive-FLMC by $74.7 \%$.

Table 6. A summary of fuzzy logic-based WSN mobility management protocols.

\begin{tabular}{cccc}
\hline Protocol & Objectives & Fuzzy Logic Metrics & Evaluation Environment \\
\hline FLMC [57] & Triggering the handoff & RSSI, link loss & $\begin{array}{c}\text { Real industrial environment } \\
\text { (refinery), simulation Cooja } \\
\text { [71] }\end{array}$ \\
MoMoRo [62] & $\begin{array}{c}\text { Extra layer to enable } \\
\text { mobility of the nodes }\end{array}$ & $\begin{array}{c}\text { Expected number of } \\
\text { transmissions (ETX), received } \\
\text { signal strength (RSS), and } \\
\text { symbol error rate (SER) variance } \\
\text { Moving state, channel condition, }\end{array}$ & Real industrial environment \\
and packet delivery & Indoor and outdoor \\
$\begin{array}{c}\text { Mobility management } \\
\text { incorporating fuzzy logic } \\
\text { for a heterogeneous IP } \\
\text { environment [66] }\end{array}$ & $\begin{array}{c}\text { Predict the node's } \\
\text { mobility and energy }\end{array}$ & $\begin{array}{c}\text { Remaining energy } \\
\text { mobility }\end{array}$ & Simulation (NS3) \\
\hline
\end{tabular}

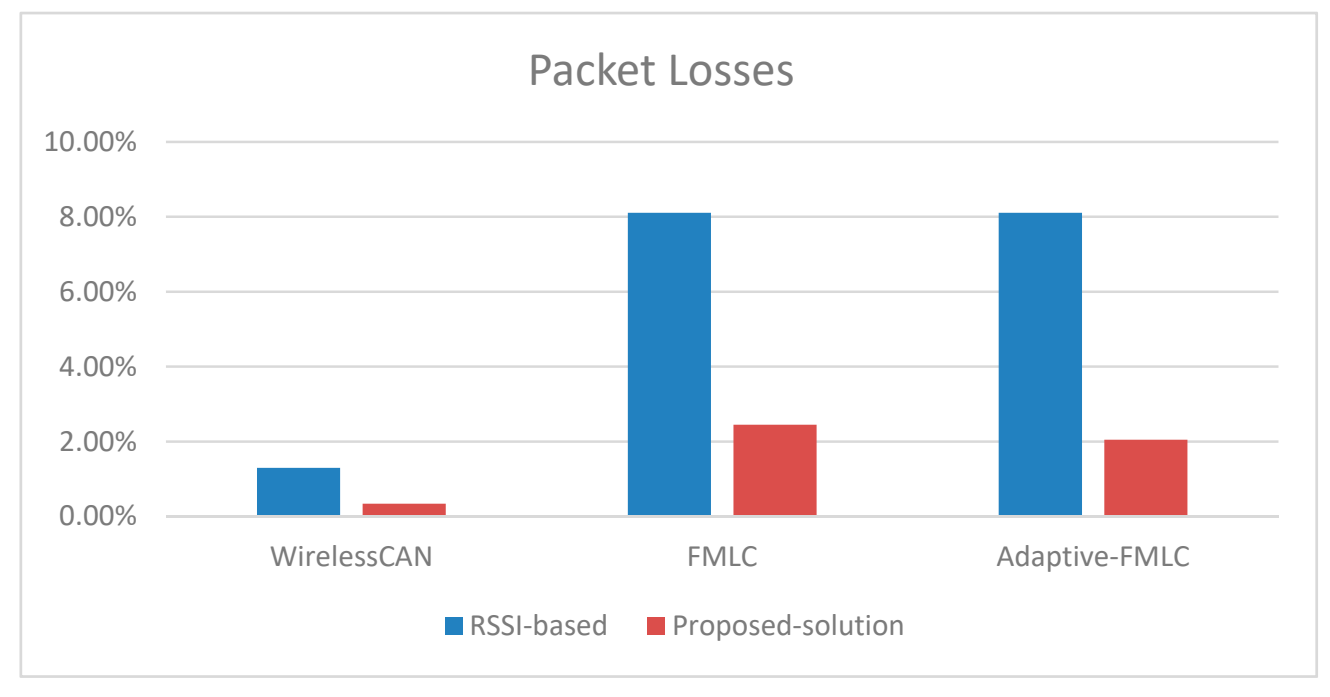

Figure 17. Packet loss comparison.

Furthermore, Figure 18 shows the number of handoffs per solution. Again, there is a comparison with the RSSI-based solution. This type of comparison shows how each solution effectively avoids the ping-pong effect. Based on the evaluation, Wireless CAN reduced the number of handoffs by $54.8 \%$, FMLC by $87 \%$, and, finally, adaptive-FLMC by $92.6 \%$. 


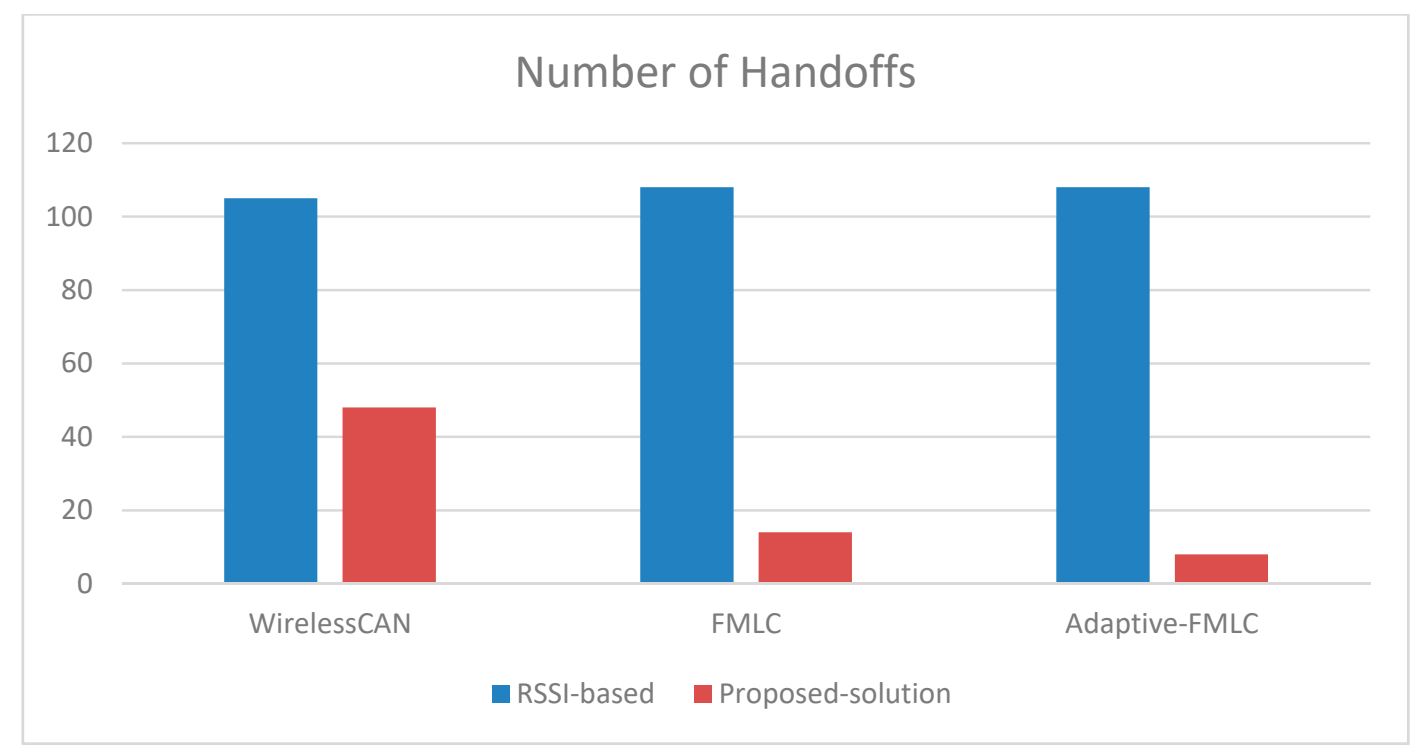

Figure 18. Number of handoffs comparison.

Finally, Table 7 depicts the percentage of power reduction of each proposed solution with an RSSI-based solution.

Table 7. Power consumption comparison with an RSSI-based solution.

\begin{tabular}{cc}
\hline Solution & Reduction Percentage \\
\hline Wireless CAN & $3.41 \%$ \\
FLMC & $8.62 \%$ \\
Adaptive-FLMC & $60.34 \%$ \\
\hline
\end{tabular}

\section{Conclusions and Future Work}

In this paper, a literature survey was presented for the most relevant related work that utilizes fuzzy logic to solve the main WSN challenges associated with the cooperative multi-robotic wireless sensor networks, namely, clustering, routing, power, and mobility management. To solve the cluster and cluster head selection process, most of proposed work presented a de-centralized approach to select the cluster head and form the clusters, where each node collects several parameters such as its residual energy, distance to the sink node, and the node's centrality, and runs fuzzy logic rules to decide whether it can be a cluster head or not. Then, on the basis of an election process, the cluster and cluster head are formulated. To solve the routing problem, the related work proposes that each node will collect certain information such as neighbouring nodes' residual energy, link quality, and distance to the sink node, and input them into the fuzzy logic system to find out the optimal route, which maximizes the packet delivery ratio, minimizes the end-to-end delay, and minimizes the nodes' energy consumption. Further, in order to optimize the nodes' energy consumption, several fuzzy-based power management and energy-saving algorithms were presented that aim at optimizing the sleep/wake cycle for the nodes and optimize the nodes' transmission efficiency by increasing the number of sink nodes and optimizing their locations concerning the other nodes. Finally, to address the mobility challenges associated with multi-robotic systems, fuzzy logic is utilized to maintain the nodes' connectivity during mobility. To address the aforementioned challenges, several input parameters were used in the fuzzy logic system such as the data, its sampling time, node remaining energy, throughput-to-workload, the number of dead nodes in the network, and the received signal strength, among others, in order to find optimal clustering, routing, and power-saving strategies, and maintain the mobile nodes' connectivity. In future work, the potential of fuzzy logic in mitigating other WSNs challenges such as interference and spectrum sharing will be investigated. Furthermore, the various approaches for 
solving CMR-WSN challenges will be compared with each other, focusing on the nodes' computational and memory resources, as well as implementation complexity and the practicality of each approach.

Author Contributions: Conceptualization, A.K.; methodology, A.K.; investigation, A.K., K.R., O.A., K.A.D., A.M.K., Z.Z.; resources, A.K., K.R., O.A., K.A.D., A.M.K., Z.Z.; writing-original draft preparation, A.K., K.R., O.A., K.A.D., A.M.K., Z.Z.; writing一review and editing, A.K., K.R., O.A., K.A.D., A.M.K., Z.Z.

Funding: This work is supported by the North Atlantic Treaty Organization (NATO) Science Peace and Security (SPS). Project title: “Hybrid Sensor Network for Emergency Critical Scenarios". Project no. SPS G4936.

Conflicts of Interest: The authors declare no conflict of interest.

\section{References}

1. Darabkh, K.A.; El-Yabroudi, M.; El-Mousa, A. BPA-CRP: A balanced power-aware clustering and routing protocol for wireless sensor networks. Ad Hoc Netw. 2019, 82, 155-171. [CrossRef]

2. Khalifeh, A.; AlQudah, M.; Tanash, R.; Darabkh, K.A. A simulation study for UAV- aided wireless sensor network utilizing Zigbee protocol. In Proceedings of the 2018 14th International Conference on Wireless and Mobile Computing, Networking and Communications (WiMob), Limassol, Cyprus, 15-17 October 2018; pp. 181-184.

3. Darabkh, K.A.; El-Yabroudi, M. A reliable relaying protocol in wireless sensor networks. In Proceedings of the 2017 IEEE European Conference on Electrical Engineering \& Computer Science, Bern, Switzerland, 17-19 November 2017; pp. 56-60.

4. Darabkh, K.A.; Alsaraireh, N. A yet efficient target tracking algorithm in wireless sensor networks. In Proceedings of the 15th IEEE Multi-conference on Systems, Signals, and Devices (SSD'18), SignalHammamet, Tunisia, 19-22 March 2018.

5. Darabkh, K.A.; Al-Jdayeh, L. A new fixed clustering based algorithm for wireless sensor networks. In Proceedings of the 14th International Wireless Communications and Mobile Computing Conference (IWCMC 2018), Limassol, Cyprus, 25-29 June 2018.

6. Darabkh, K.A.; Al-Rawashdeh, W.; Hawa, M.; Saifan, R.; Khalifeh, A. A novel clustering protocol for wireless sensor networks. In Proceedings of the 2017 International Conference on Wireless Communications, Signal Processing and Networking (WiSPNET 2017), Chennai, India, 22-24 March 2017.

7. Darabkh, K.A.; N Al-Maaitah, I.J.; Khalifeh, A. EA-CRP: A novel energy-aware clustering and routing protocol in wireless sensor networks. Coput. Electr. Eng. 2018, 72, 702-718. [CrossRef]

8. Darabkh, K.A.; Muqat, R. An efficient protocol for minimizing long-distance communications over wireless sensor networks. In Proceedings of the 15th IEEE Multi-conference on Systems, Signals, and Devices (SSD'18), Hammamet, Tunisia, 19-22 March 2018.

9. Arai, T.; Pagello, E.; Parker, L.E. Guest editorial advances in multi robot systems. IEEE Trans. Robot. Autom. 2002, 18, 655-661. [CrossRef]

10. Vergnano, A.; Thorstensson, C.; Lennartson, B.; Falkman, P.; Pellicciari, M.; Leali, F.; Biller, S. Modeling and optimization of energy consumption in cooperative multi-robot systems. IEEE Trans. Autom. Sci. Eng. 2012, 9, 423-428. [CrossRef]

11. Tuna, G.; Gulez, K.; Gungor, V.C. Communication related design considerations of WSN-aided multi-robot SLAM. In Proceedings of the 2011 IEEE International Conference on Mechatronics, Istanbul, Turkey, 13-15 April 2011; pp. 493-498.

12. Meng, Y.; Gan, J. A distributed swarm intelligence based algorithm for a cooperative multi-robot construction task. In Proceedings of the 2008 IEEE Swarm Intelligence Symposium, St. Louis, MO, USA, 21-23 September 2008; pp. 1-6.

13. Tuna, G.; Gungor, V.; Gulez, K. An autonomous wireless sensor network deployment system using mobile robots for human existence detection in case of disasters. Ad Hoc Netw. 2014, 13, 54-68. [CrossRef]

14. Khalifeh, A.; Al-Agtash, S.; Tanash, R.; AlQudah, M. Deploying agents for monitoring and notification of wireless sensor networks. In Proceedings of the 2016 IEEE 28th International Conference on Tools with Artificial Intelligence (ICTAI), San Jose, CA, USA, 6-8 November 2016; pp. 754-757. 
15. Khalifeh, A.; Bartolini, N.; Silvestri, S.; Bongiovanni, G.; Al-Assaf, A.; Alwardat, R.; Alhaj-Ali, S. Hybrid wireless sensor networks: A prototype. In Proceedings of the 2019 IFIP Conference on Human-Computer Interaction, Paphos, Cyprus, 2-6 September 2019; Springer: Cham, Switzerland; pp. 549-553.

16. Sayyed, A.; de Araújo, G.; Bodanese, J.; Becker, L. Dual-stack single-radio communication architecture for UAV acting as a mobile node to collect data in WSNs. Sensors 2015, 15, 23376-23401. [CrossRef]

17. Darabkh, K.A.; Odetallah, S.; Al-Qudah, Z.; Khalifeh, A.; Shurman, M. Energy-aware and density-based clustering and relaying protocol (EA-DB-CRP) for gathering data in wireless sensor networks. Appl. Soft Comput. 2019, 80, 154-166. [CrossRef]

18. Bagci, F.; Khalifeh, A. Cluster communication protocol for wireless sensor networks. Int. J. Sens. Netw. 2016, 20,84-91. [CrossRef]

19. Velimirovic, A.S.; Djordjevic, G.L.; Velimirovic, M.M.; Jovanovich, M.D. Fuzzy ring-overlapping range-free (FRORF) localization method for wireless sensor networks. Comput. Commun. 2012, 35, 1590-1600. [CrossRef]

20. Zhao, L.; Wen, X.; Li, D. Amorphous localization algorithm based on BP artificial neural network. In Proceedings of the International Conference on Software Intelligence Technologies and Applications \& International Conference on Frontiers of Internet of Things 2014, Hsinchu, Taiwan, 2014; pp. 178-183.

21. Lin, C.; Mon, Y.; Lee, C.; Juang, J.; Rudas, I. ANFIS-based indoor location awareness system for the position monitoring of patients. Acta Polytech. Hung. 2014, 11, 37-48.

22. Liu, Z.-K.; Zhong, L. Node self-localization algorithm for wireless sensor networks based on modified particle swarm optimization. In Proceedings of the 27th Chinese Control and Decision Conference (2015 CCDC), Qingdao, China, 2015; pp. 5968-5971.

23. Chagas, S.H.; Martins, J.B.; de Oliveira, L.L. An approach to localization scheme of wireless sensor networks based on artificial neural networks and Genetic Algorithms. In Proceedings of the 10th IEEE International NEWCAS Conference, Montreal, QC, USA, 2012; pp. 137-140.

24. Krishnaprabha, R.; Gopakumar, A. Performance of gravitational search algorithm in wireless sensor network localization. In Proceedings of the 2014 IEEE National Conference on Communication, Signal Processing and Networking (NCCSN), Palakkad, India, 2014; pp. 1-6.

25. Kulkarni, R.V.; Venayagamoorthy, G.K. Bio-inspired algorithms for autonomous deployment and localization of sensor nodes. IEEE Trans. Syst. Man Cybern. Part C 2010, 40, 663-675. [CrossRef]

26. McBratney, A.; Inakwu, O. Application of fuzzy sets in soil science: Fuzzy logic, fuzzy measurements and fuzzy decisions. Geoderma 1997, 77, 85-113. [CrossRef]

27. Darabkh, K.A.; Alsukour, O. Novel protocols for improving the performance of ODMRP and EODMRP over mobile ad hoc networks. Int. J. Distrib. Sens. Netw. 2015, 2015, 1-18. [CrossRef]

28. Zimmermann, H. Fuzzy Set Theory and its Applications; Kluwer Academic Publications: Boston, MA, USA, 2001.

29. Maksimović, M.; Vujović, V.; Milošević, V. Fuzzy logic and wireless sensor networks-A survey. J. Intell. Fuzzy Syst. 2014, 27, 877-890. [CrossRef]

30. Mizutani, E.; Sun, C.; Jang, R.; Roger, J. Neuro-Fuzzy and Soft Computing: A Computational Approach to Learning and Machine Intelligence; Prentice Hall: Upper Saddle River, NJ, USA, 1997.

31. Kim, J.; Park, S.; Han, Y.; Chung, T. CHEF: Cluster head election mechanism using fuzzy logic in wireless sensor networks. In Proceedings of the 2008 10th IEEE International Conference on Advanced Communication Technology, Gangwon-Do, Korea, 17-20 February 2008; Volume 1, pp. 654-659.

32. Handy, M.; Haase, M.; Timmermann, D. Low energy adaptive clustering hierarchy with deterministic cluster-head selection. In Proceedings of the 4th IEEE International Workshop on Mobile And Wireless Communications Network, Stockholm, Sweden, 9-11 September 2002; pp. 368-372.

33. Hamzah, A.; Al-Jarrah, M.S.O.; Taqieddin, E. Energy-efficient fuzzy-logic-based clustering technique for hierarchical routing protocols in wireless sensor networks. Sensors 2019, 19, 561. [CrossRef]

34. Thangaramya, K.; Kulothungan, K.; Logambigai, R.; Selvi, M.; Ganapathy, S.; Kannan, A. Energy aware cluster and neuro-fuzzy based routing algorithm for wireless sensor networks in IoT. Comput. Netw. 2019, 151, 211-223. [CrossRef]

35. Nguyen, T.T.; Pan, J.; Chu, S.; Dao, T.; Do, V. Improved performance of wireless sensor network based on fuzzy logic for clustering scheme. In Proceedings of the International Conference on Smart Vehicular Technology, Transportation, Communication and Applications, Mount Emei, China, 25-28 October 2018; pp. 104-113. 
36. Nayak, P.; Devulapalli, A. A fuzzy logic-based clustering algorithm for WSN to extend the network lifetime. IEEE Sens. J. 2015, 16, 137-144. [CrossRef]

37. Vajdi, A.; Zhang, G.; Wang, Y.; Wang, T. A new self-management model for large-scale event-driven wireless sensor networks. IEEE Sens. J. 2016, 16, 7537-7544. [CrossRef]

38. Toloueiashtian, M.; Motameni, H. A new clustering approach in wireless sensor networks using fuzzy system. J. Supercomput. 2018, 74, 717-737. [CrossRef]

39. Qin, J.; Fu, W.; Gao, H.; Zheng, W.X. Distributed K-means algorithm and fuzzy C-means algorithm for sensor networks based on multi-agent consensus theory. IEEE Trans. Cyber. 2016, 47, 772-783. [CrossRef]

40. Faramondi, L.; Oliva, G.; Setola, R.; Hadjicostis, C.N. Distributed C-Means clustering via broadcast-only token-passing. IEEE Trans. Control Netw. Syst. 2019. [CrossRef]

41. Gasparri, A.; Oliva, G. Fuzzy Opinion Dynamics. In Proceedings of the American Control Conference 2012 (ACC2012), Montreal, QC, Canada, 27-29 June 2012; pp. 5640-5645.

42. Oliva, G.; la Manna, D.; Fagiolini, A.; Setola, R. Distributed data clustering via opinion dynamics. Int. J. Distrib. Sens. Netw. 2015, 11, 753102. [CrossRef]

43. Mothku, S.; Rout, R. Adaptive fuzzy-based energy and delay-aware routing protocol for a heterogeneous sensor network. J. Comput. Netw. Commun. 2019. [CrossRef]

44. Hu, X.; Ma, L.; Ding, Y.; Xu, J.; Li, Y.; Ma, S. Fuzzy logic-based geographic routing protocol for dynamic wireless sensor networks. Sensors 2019, 19, 196. [CrossRef] [PubMed]

45. Gholipour, M. A new ant colony optimization routing approach based fuzzy clustering in wireless sensor network. Rev. Pub. 2019, 5, 469-479.

46. Sert, S.; Alchihabi, A.; Yazici, A. A Two-tier distributed fuzzy logic based protocol for efficient data aggregation in multi-hop wireless sensor networks. IEEE Trans. Fuzzy Syst. 2018, 26, 3615-3629. [CrossRef]

47. Tabatabaei, S.; Rajaei, A.; Rigi, A.M. A novel energy-aware clustering method via Lion Pride Optimizer Algorithm (LPO) and fuzzy logic in wireless sensor networks (WSNs). Wirel. Pers. Commun. 2019, 108, 1-23. [CrossRef]

48. Koosheshi, K.; Ebadi, S. Optimization energy consumption with multiple mobile sinks using fuzzy logic in wireless sensor networks. Wirel. Netw. 2019, 25, 1215-1234. [CrossRef]

49. Balaji, S.; Julie, E.G.; Robinson, Y.H. Development of fuzzy based energy efficient cluster routing protocol to increase the lifetime of wireless sensor networks. Mob. Netw. Appl. 2019, 24, 394-406. [CrossRef]

50. Collotta, M.; Pau, G.; Salerno, V.M.; Scatà, G. A fuzzy based algorithm to manage power consumption in industrial Wireless Sensor Networks. In Proceedings of the 2011 9th IEEE International Conference on Industrial Informatics, Lisbon, Portugal, 26-29 July 2011; pp. 151-156.

51. Collotta, M.; Pau, G.; Scatá, G. A fuzzy system to reduce power consumption in wireless sensor networks: A comparison between WirelessHART and IEEE 802.15. 4. In Proceedings of the 2014 IEEE International Energy Conference (ENERGYCON), Dubrovnik, Croatia, 13-16 May 2014; pp. 766-771.

52. Pau, G. Power consumption reduction for wireless sensor networks using a fuzzy approach. Int. J. Eng. Technol. Innov. 2016, 6, 55-67.

53. Masdari, M.; Naghiloo, F. Fuzzy logic-based sink selection and load balancing in multi-sink wireless sensor networks. Wirel. Pers. Commun. 2017, 97, 2713-2739. [CrossRef]

54. Collotta, M.; Pau, G.; Maniscalco, V. A fuzzy logic approach by using particle swarm optimization for effective energy management in IWSNs. IEEE Trans. Ind. Electron. 2017, 64, 9496-9506. [CrossRef]

55. Cao, C.; Zhu, X. Energy management using optimal fuzzy logic control in wireless sensor network. Int. J. Online Eng. 2018, 14, 35-52. [CrossRef]

56. Pau, G.; Salerno, V.M. Wireless Sensor Networks for Smart Homes: A Fuzzy-based solution for an energy-effective duty cycle. Electronics 2019, 8, 131. [CrossRef]

57. Zinonos, Z.; Chrysostomou, C.; Vassiliou, V. Wireless sensor networks mobility management using fuzzy logic. Ad Hoc Networks 2014, 16, 70-87. [CrossRef]

58. Silva, R.; Zinonos, Z.; Silva, J.S.A.; Vassiliou, V. Mobility in WSNs for critical applications. In Proceedings of the 2011 IEEE Symposium on Computers and Communications (ISCC), Kerkyra, Greece, 28 June-1 July 2011; pp. 451-456.

59. Zinonos, Z.; Chrysostomou, C.; Vassiliou, V. Controlling the handoff procedure in an oil refinery environment using fuzzy logic. In Proceedings of the 2012 IEEE 15th International Conference on Computational Science and Engineering, Nicosia, Cyprus, 5-7 December 2012; pp. 477-483. 
60. Zinonos, Z.; Silva, R.; Vassiliou, V.; Silva, J. Mobility solutions for wireless sensor and actuator networks with performance guarantees. In Proceedings of the 18th International Conference on Telecommunications, Ayia Napa, Cyprus, 8-11 May 2011; pp. 406-411.

61. Zinonos, Z.; Vasilios, V. S-GinMob: Soft-handoff solution for mo-bile users in industrial environments. In Proceedings of the 2011 International Conference on Distributed Computing in Sensor Systems and Workshops (DCOSS), Barcelona, Spain, 27-29 June 2011; pp. 1-6.

62. Ko, J.; Chang, M. MoMoRo: Providing mobility support for low-power wireless applications. IEEE Syst. J. 2015, 9, 585-594. [CrossRef]

63. Ma, J.; Yang, D.; Zhang, H.; Gidlund, M. A reliable handoff mechanism for mobile industrial wireless sensor networks. Sensors 2017, 17, 1797. [CrossRef]

64. Katkar, P.S.; Ghorpade, V.R. Fuzzy approach to predict mobility and energy to prolong the life of Wireless sensor network. In Proceedings of the 2016 IEEE International WIE Conference on Electrical and Computer Engineering (WIECON-ECE), Pune, India, 19-21 December 2016; pp. 12-15.

65. Zhang, W. Handover decision using fuzzy MADM in heterogeneous networks. In Proceedings of the 2004 IEEE Wireless Communications and Networking Conference (IEEE Cat. No.04TH8733), Atlanta, GA, USA, 21-25 March 2004; pp. 653-658.

66. Chan, M.L.; Sheriff, R.E.; Hu, Y.F.; Conforto, P.; Tocci, C. Mobility management incorporating fuzzy logic for heterogeneous a IP environment. IEEE Commun. Mag. December 2001, 39, 42-51. [CrossRef]

67. Liao, H.; Tie, L.; Du, Z. A vertical handover decision algorithm based on fuzzy control theory. In Proceedings of the First International Multi-Symposiums on Computer and Computational Sciences, Hangzhou, China, 20-24 June 2006; Volume 2, pp. 309-313.

68. Barolli, L.; Durresi, A.; Xhafa, F.; Koyama, A. A Fuzzy-based handover system for wireless cellular networks: A case study for handover enforcement. In International Conference on Network-Based Information Systems; Takizawa, M., Barolli, L., Enokido, T., Eds.; Springer: Berlin/Heidelberg, Germany, 2008.

69. Zinonos, Z.; Vassiliou, V.; Christodoulou, K. Reliable Mobility Support for e-Health Monitoring: A Performance Evaluation. In Proceedings of the 2018 Innovations in Intelligent Systems and Applications (INISTA), Thessaloniki, Greece, 3-5 July 2018; pp. 1-6.

70. Zinonos, Z.; Vassiliou, V.; Chrysostomou, C. Adaptive fuzzy logic mobility management for WSN. In Proceedings of the 2014 IEEE International Conference on Distributed Computing in Sensor Systems, Marina Del Rey, Marina Del Rey, CA, USA, 26-28 May 2014; pp. 302-307.

71. Zinonos, Z.; Vassiliou, V.; Christofides, T. Radio propagation in industrial wireless sensor network environments: From testbed to simulation evaluation. In Proceedings of the 7th ACM Workshop on Performance Monitoring and Measurement of Heterogeneous Wireless and Wired Networks, Paphos, Cyprus, 21 October 2012; pp. 125-132. 